\title{
A kinematic study of skilled reaching movement in rat
}

\author{
Pierantonio Parmiani ${ }^{\mathrm{a}, \mathrm{b}}$, Cristina Lucchetti ${ }^{\mathrm{c}}$, Claudio Bonifazzi ${ }^{\mathrm{a}}$, Gianfranco Franchi ${ }^{\mathrm{a}}$ \\ a Department of Biomedical and Specialty Surgical Sciences, Section of Human Physiology, University of Ferrara, 44121, Ferrara, Italy \\ b Center for Translational Neurophysiology, Istituto Italiano di Tecnologia, 44121, Ferrara, Italy \\ ${ }^{c}$ Department of Biomedical, Metabolic and Neural Sciences, Section of Physiology and Neuroscience, University of Modena and Reggio Emilia, 43125, Modena, Italy
}

\section{A R T ICLE INFO}

\section{Keywords}

Motion tracking system

Skilled reaching

Kinematic variables

Rat

\begin{abstract}
A B S T R A C T
Background: In the rat, the single-pellet reaching task includes orienting, reaching, grasping and retracting movements. It has previously been described by notation techniques, high-speed video and cineradiographic recordings. Recently, high-definition cameras have been used to track paw and digit movements with DeepLabCut, a machine-learning algorithm for markerless estimation of paw position.

New method: Our new approach consists of positioning three high-speed infrared digital cameras to track the full motion of markers on the rat's body. This provided a previously unavailable 3D recording of skilled reaching kinematics in the rat moving freely in the reaching box, which were analysed by Qualisys Track Manager software and MATLAB.

Results: This method enabled description of kinematic parameters unobtainable without motion tracking and provided insight into the spatiotemporal metrics of movements used to perform skilled reaching. It revealed that orientation features three steps and reaching has two bimodal start-point distributions, one along the horizontal axis and one along the vertical axis. At the end of reaching, the wrist/paw occupies the same position as the nose at the end of orienting. In grasping, averaging trajectories confirmed the marker lowering and target approaching.

Comparison with existing methods: Our method required significantly reduced time to label data and obviates the need for off-line manual marking of videos. It provides an efficient means of capturing volumes containing the entire range of marker movements.

Conclusions: This study validated a new and efficient approach for quantifying rat movement kinematics, useful for comparing preclinical and clinical conditions.
\end{abstract}

\section{Introduction}

Skilled reaching, the act of reaching to grasp an object as occurs in a reach-to-eat task, shares many similarities among both primates and rodents (Whishaw and Pellis, 1990; Whishaw, 1996). In rodents, skilled reaching to eat is a learned and composite movement in which the rat, inside a reaching box, uses olfactory cues, proprioception, whiskers, and tactile nose sense to locate food, and to define a reaching path for the paw to target the pellet (Whishaw and Tomie, 1989; Whishaw et al., 2017; Parmiani et al., 2018). Skilled reaching task starts with orienting, which consists of the rat's approach to the front wall of the reaching box, and nose positioning over the pellet. Orienting is followed by reaching, i.e. transporting the paw towards the pellet; grasping, which involves prehension of the pellet; and paw retraction, to bring the pellet to the mouth (Alaverdashvili et al., 2008).
The rat's skilled reaching behaviour emerges as an important model for investigating many topics in neurosciences research, from motor behaviour (Whishaw and Tomie, 1989; Alaverdashvili et al., 2008) to sensorimotor integration (Whishaw and Pellis, 1990; Hermer-Vazquez et al., 2007; Parmiani et al., 2018), or to study neural mechanisms of movement (Azim et al., 2014) and also serves as a model for studying many motor system injuries that affect motor behaviour (Klein et al., 2012; Viaro et al, 2017).

Skilled reaching has been previously described in rats by means of notation techniques (Whishaw et al., 1992; Gharbawie and Whishaw, 2006), video (Whishaw et al., 2008), as well as cineradiographic recordings (Alaverdashvili et al., 2008). More recently, high-speed single (Wong et al., 2015; Ellens et al., 2016; Nica et al., 2018; Parmiani et al., 2018) and multiple cameras have respectively been used in kinematic studies to reconstruct reaching trajectories (Azim et al., 2014; Mathis et al., 2018; Bova et al., 2019). The most recent innovation for describing/analysing rat reaching, how-

* Corresponding authors at: Department of Biomedical and Specialty Surgical Sciences, Section of Human Physiology, University of Ferrara, via Fossato di Mortara 17-19, 44100, Ferrara, Italy.

E-mail addresses: pierantonio.parmiani@unife.it (P. Parmiani); fhg@unife.it (G. Franchi) 
ever, includes a machine-learning algorithm to enable digitization of joint segments from markerless video recordings (Mathis et al., 2018; Bova et al., 2019).

We, on the other hand, used an infrared three-dimensional motion-tracking system, which, unlike the machine-learning approach, does not required significant time and effort to manually mark videos, coupled with behavioural video-recordings. Our primary goal was to characterize head and forelimb movement coordination in a single-pellet reaching task inside a reaching box, where the animal can freely reposition its body. In detail, we set up 3 digital cameras that precisely captured the 3D motion of all markers attached to the rat body, in a capture volume containing the entire expected range of markers movements. The expectation was that this approach would reveal insight into the spatiotemporal metrics of the kinematics of movements used to perform orienting and reaching-grasping movements.

\section{Materials and methods}

\subsection{Subjects and ethical approval}

The subjects were five male albino Wistar rats, each weighing 280-330 g, raised in the animal house at the University of Ferrara, Italy. The experimental plan was designed in compliance with Italian law regarding the care and use of experimental animals (DL 26/2014) and approved by both the institutional review board of the University of Ferrara (OBA) and the Italian Ministry of Health.

\subsection{Feeding and food restriction}

Rats were housed in polycarbonate cages $(53 \mathrm{~cm}$ long, $37 \mathrm{~cm}$ wide, and $21 \mathrm{~cm}$ deep) with sawdust bedding, in groups of three or four to a colony, under a $12 \mathrm{~h}: 12 \mathrm{~h}$ light/dark cycle with light starting at 07:30 h. All testing and training was performed at the same time of day, during the light phase of the cycle. The animals received water ad libitum. They were food-deprived before the training started, and maintained about $90 \%$ of their initial body weight throughout the experiment. The week before training began, each rat received twenty banana-flavoured round food pellets (Rodent Tab 45 mg, AIN-76A, TestDiet, Richmond, USA) $1 \mathrm{~h}$ prior to the daily fodder ration. The pellets would serve as reaching targets in a single-pellet reaching box. To maintain body weight, the rats were given an additional amount of food in their home cages at least $1 \mathrm{~h}$ after the end of the training or testing session.

\subsection{Reaching box and single-pellet training}

The reaching box was similar to that described by Metz and Alaverdashvili, (Metz et al. 2000; Alaverdashvili et al., 2008) and is made of clear plexiglas $(340 \times 390 \times 134 \mathrm{~mm}$ wide). In the middle of the front wall, a 10 -mm wide vertical opening allows the animal to reach for pellets placed on a shelf. The shelf, $15 \mathrm{~mm}$ wide and $20 \mathrm{~mm}$ long, is attached outside of the front wall and positioned $30 \mathrm{~mm}$ above the floor. On the upper side of the shelf and aligned to the midline of the box, there is a round indentation (diameter $7 \mathrm{~mm}$, depth $2.5 \mathrm{~mm}$, and distance from the front wall $10 \mathrm{~mm}$ ) for food pellet positioning.

During pre-training (about one week), the rat was placed in the box for 20-minute daily sessions, during which it was allowed to explore the reaching box, and some food pellets were placed on the shelf to promote reaching-grasping through the slot. Pre-training ended when the rat started to reach for the food pellet. Training proper consisted of daily sessions lasting $20 \mathrm{~min}$, during which the rat learned to grasp the pellet with the preferred paw. Paw preference was established when at least $60 \%$ of a minimum of 10 reach attempts were made using the left or right forelimb. The rat was taught to advance from the posterior part of the box to the front wall, to sniff for the pellet on the shelf, and to perform the reaching-grasping sequence only if the pellet was present. Otherwise, the rat had to go back to the posterior part of the box to start another trial. In order to facilitate learning of this movement sequence, a food pellet was dropped in the posterior part of the box in the first training sessions. The success level of grasping was scored in the last week of training and during movement recording. At the end of each session, the success rate reached by each rat was computed as the ratio between the grasping movements in which the rat brought a food pellet to its mouth, and the total number of prehensions in the session multiplied by 100 . For each rat, training ended after about three weeks, when the percentage success rate was around $50 \%$ of total trials.

\subsection{Video-recording of rat movements}

The rat was video-recorded for the entire duration of the recording session at 200 frames/s, using a JVC GC-PX100 camera with a resolution of $640 \times 360$ pixels. The recording videocamera was positioned so as to obtain a lateral view of the animal inside the box, ipsilateral to the preferred paw (Fig. 1, left frames: A-E) and was used as a reference to clarify the marker positions for the 3D kinematic analysis (Fig. 1, right frames: A1-E1). Each trial was composed of the following sequence of movements: A/A1: approaching, i.e. walking to the front wall of the box; $\mathrm{B} / \mathrm{B} 1$ : orienting, when the head was raised from the floor until the nose poked into the slot; $\mathrm{C} / \mathrm{C} 1$ : reaching, i.e. advancing forelimb through the slot towards the pellet; D/D1: grasping the pellet on the shelf; and E/ E1: retracting the grasped food for eating. Videotaped movements were analysed frame-by-frame using Avidemux 2.6 software (www.avidemux. org) (Parmiani et al., 2018).

\subsection{Kinematic recording of movements}

Rat movements were recorded and measured using an infrared three-dimensional motion-tracking system (Qualisys Motion Capture System; Qualisys North America Inc, Charlotte, USA) (Bonazzi et al., 2013). Qualisys uses an Automatic Identification of Markers (AIM) model which is basically a marker classifier which learns from each trial it tracks. What this means is that after labelling each marker, it updates the AIM model. When a new file is opened, AIM model is applied so the Qualisys automatically labels the markers over the all trial and automatically filling in gaps of certain sizes. Four adhesive infrared-reflective spheres (diameter $4 \mathrm{~mm}$ and weight $0.09 \mathrm{~g}$ ) were affixed to the rat's body for use as markers (Fig. 2A). In all recording sessions, markers were placed by the same operator so as to minimize both variability in their position and upsetting the animal. Rats initially attempted to remove the markers, but then got used to their presence, with the exception of the digit marker; all rats reacted badly to this marker, and, out of five rats, only three tolerated its presence. Hence, we were forced to record a smaller number of trials with the marker on the digit.

The 3D system used for tracking the markers is equipped with three infrared cameras, placed around the reaching box (Fig. 2B). Cameras were adjusted to create a volume that is viewed by all three cameras (the capture volume) and contains the entire expected range of marker movement. Indeed, each marker must be seen by all cameras throughout the movement to have a correct 3D recording (fill level: 100\%). Cameras were positioned at a distance of about $90 \mathrm{~cm}$ from the platform (on which the reaching box rests) and $10 \mathrm{~cm}$ above it, resulting in a viewing angle of about $20^{\circ}$. Two cameras were positioned lateral to the preferred paw, and the third camera was positioned in front of the pellet to be reached for (Fig. 2B). The cameras were calibrated according to the Qualisys Motion Capture Analysis System protocol, placing a stationary L-shaped reference structure with 4 markers to define the origin and orientation of the 3D-coordinates system. The directions of 


\section{Video Frames}
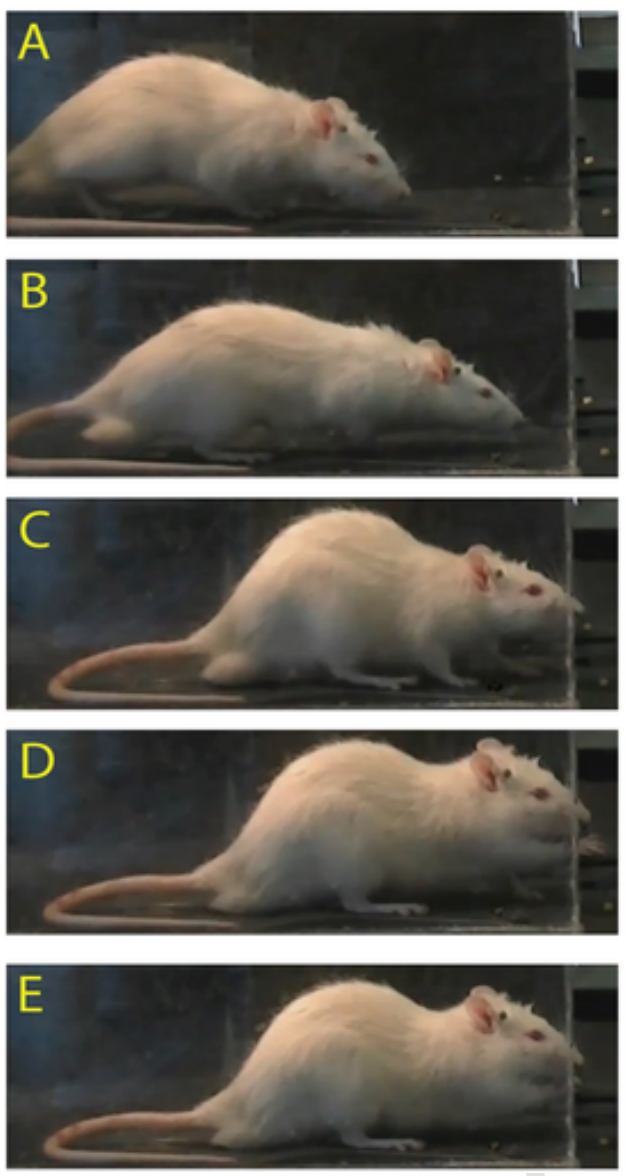

\section{3-D Tracking System Frames}
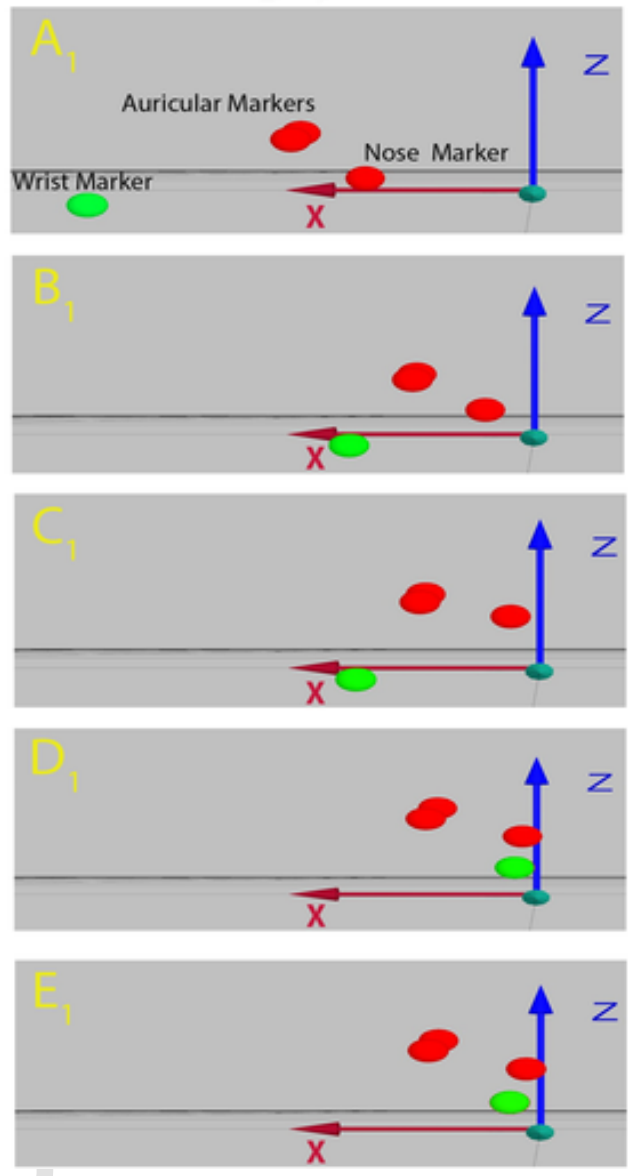

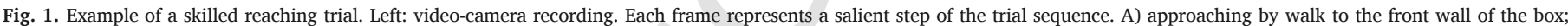

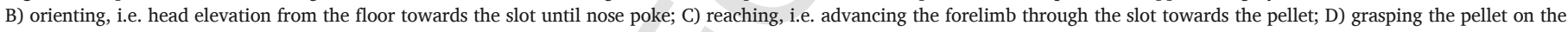

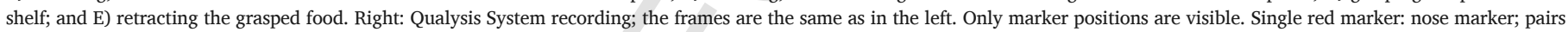
of red markers: auricular markers; green marker: wrist marker.

$\mathrm{X}$-, Y- and Z-axis coordinates were horizontal, depth and vertical, respectively. The pellet reference position was defined before each recording session by placing a marker in the indentation of the reaching box shelf (Fig. 2C). Each kinematic recording was triggered by the experimenter when the rat began to approach the front wall, and lasted $5 \mathrm{~s}$ to include a single full trial (mean trial duration $\sim 1 \mathrm{~s}$ ). Light from two LEDs, one visible and the other infrared, positioned within the visual field of the cameras, was switched on by the experimenter upon triggering the kinematic recording, thereby allowing synchronization of the two recording systems. Movements were recorded with a $100-\mathrm{Hz}$ sampling rate, and kinematic features were analysed off-line by Qualisys Track Manager software and custom MATLAB scripts (The MathWorks Inc., MA, USA). The trials in which all markers were not clearly recorded throughout the whole duration (fill level $<100 \%$ ) were not considered in the off-line analysis; for this reason the number of analysed trials was fewer than those recorded, and was different for each rat.

In order to record movement kinematics, four markers were positioned on each rat's body using hot glue as follows: the first two markers near the medial auricular border, the third on the nose $(3-4 \mathrm{~mm}$ from the tip) and the fourth either on the lateral border of the wrist or the last phalangeal joint of the two middle digits, Fig. 2A). The auricular markers were used only as a reference to unequivocally identify the nose marker during head movements: in this way the nose marker enabled record of orienting kinematics. The fourth marker, depending on its position, detects either limb or paw movements, and enables record- ing of the reaching or grasping kinematics, respectively. It was not possible to place the markers simultaneously on both wrist and digit, since the Qualisys system is unable to distinguish between very close markers when the rat is moving, especially when the forelimb goes through the slot during a reaching movement. Hence, wrist and digit markers were applied in different blocks of trials, meaning that reaching and grasping movements were recorded in different sessions, and are consequently reported in different Figures (Figs. 3 and 4 respectively). The markers applied to the rat's body did not interfere with the movements, except the marker applied to the digits, which reduced the successful pellet-grasping score to $<50 \%$.

\subsection{Movement pattern analysis}

Taking into account the sequence of movements that compose the trial (Fig. 1), in our analysis we considered the movements orienting, reaching and grasping. Orienting and reaching analyses were carried out on five rats, while grasping analysis on three rats due to the intolerance of the other two for the markers. We were unable to study the retract movement itself, since, in its final part, the wrist marker was not visible to all cameras; however, we referred to the beginning of retract, in which the wrist or digit marker was visible, to define the end of grasping. We then analysed orienting kinematics using the nose marker, reaching kinematics using the wrist marker, and grasping kinematics using the digit marker, as each marker provides an optimal de- 


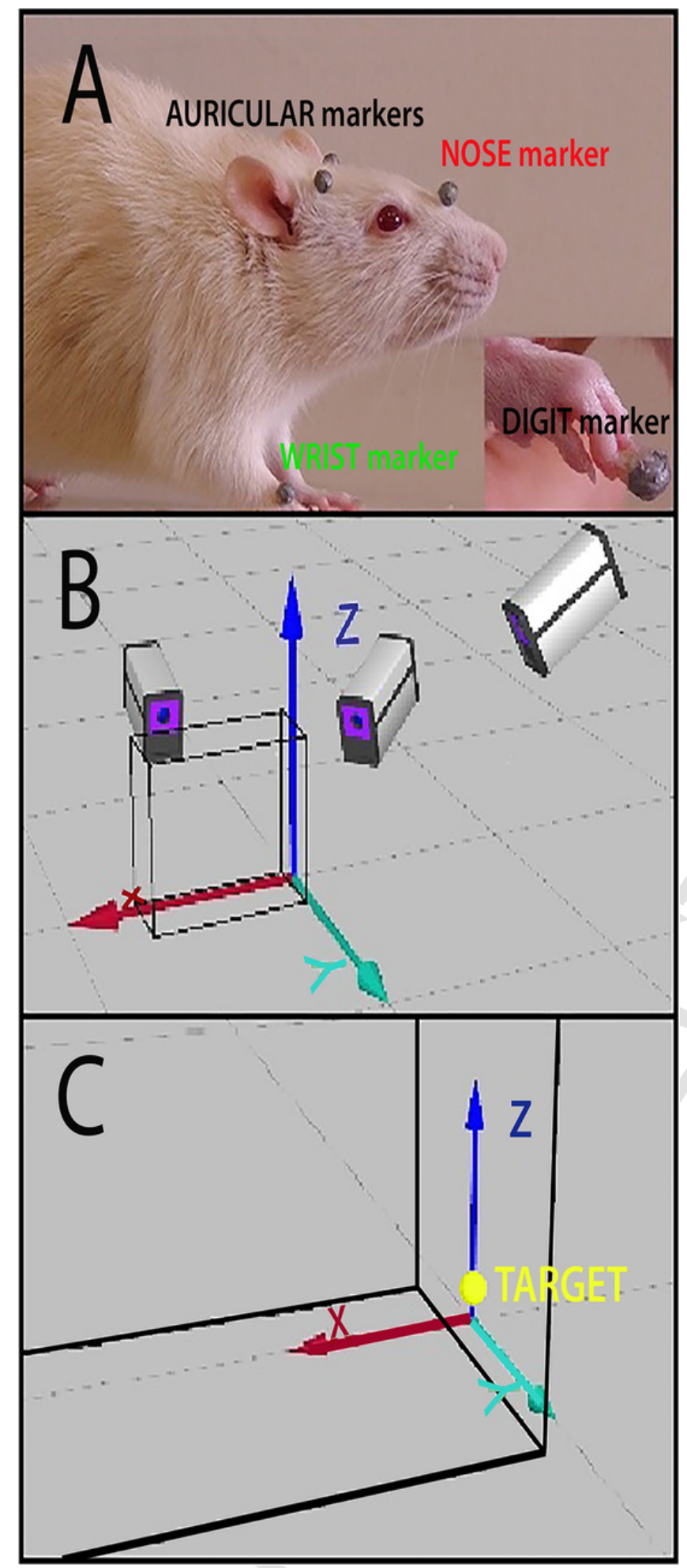

Fig. 2. Qualisys System setup. A) marker positions on the rat's body. B) Qualisys camera positions with respect to the reaching box. C) 3D-coordinates system origin $(0,0,0)$ and axes orientation (horizontal: X, depth: Y, vertical: Z) and versus (arrows direction indicates positive values) as obtained by Qualisys calibration.

scription of the velocity peak, thereby characterizing the individual movements (Figs. 3 and 4).

Fig. 3A shows an example of the nose (red) and wrist (green) marker trajectories along the horizontal and vertical axes; individual trials were split off-line into a sequence of three movements, namely,
A 2-D Markers Trajectories
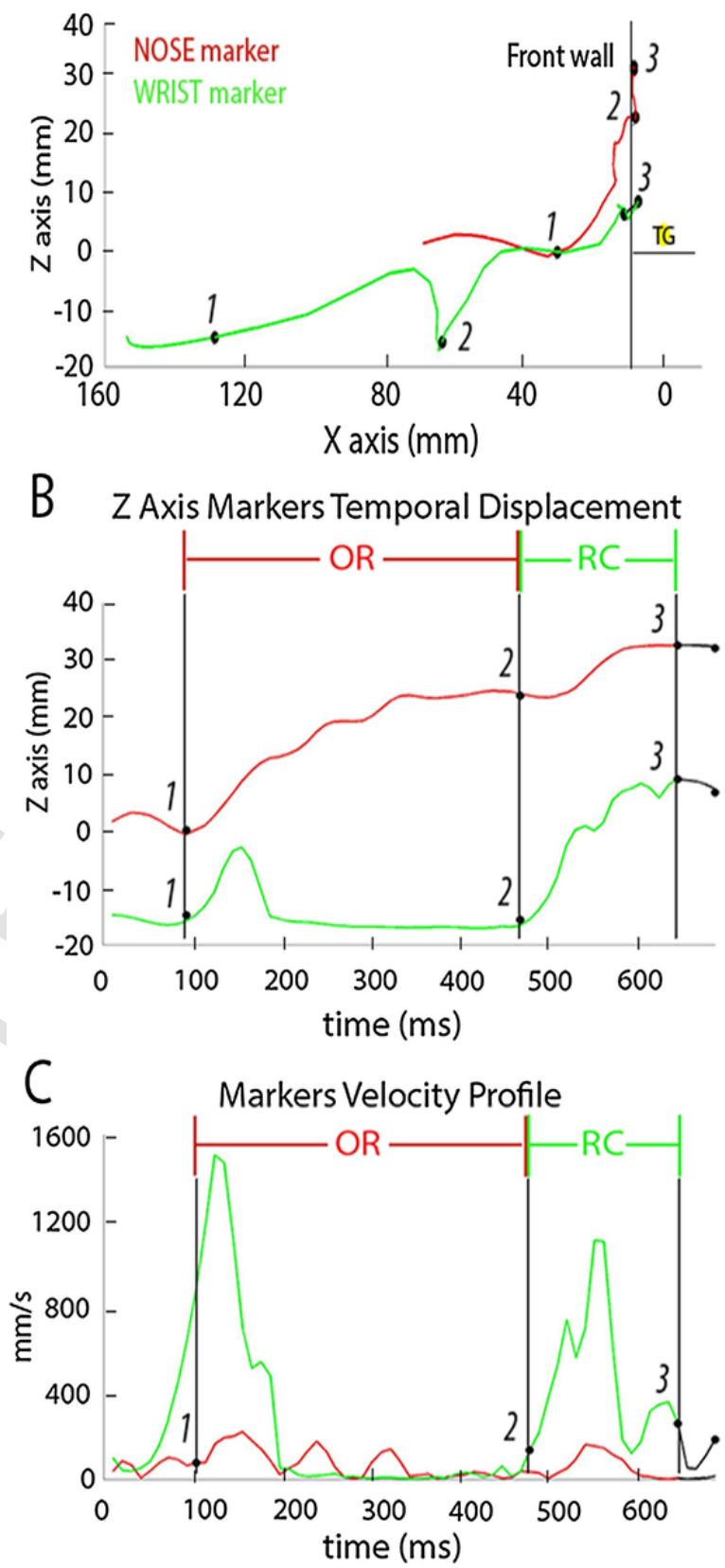

Fig. 3. Example of nose and wrist marker trajectories in the same trial presented in Fig. 1 , as recorded by Qualisys and split off-line. The figure shows the nose and wrist marker trajectories recorded during the task, while the animal in the box approaches the front wall from the left towards the target (pellet) to the right. Black dots and the corresponding numbers mark the start and the end of movements, as defined in the Methods section. OR: orienting movement; RC: reaching movement. A) X axis: horizontal axis; Z axis: vertical axis; $\mathrm{X}$ and $\mathrm{Z}$ axes 0 corresponded to target position. Nose trajectory in red, wrist trajectory in green, target (TG, pellet) in yellow. The vertical line represents the front wall of the box, and the small horizontal line below the TG represents the shelf. Numbers: 1 , orienting start point; 2 , orienting end point/reaching start point; and 3) reaching end point. The wrist marker does not show a clear velocity peak during grasping (see rightmost interval after point 3 in Fig. 3C). For this reason, the grasping kinematics were not derived from the wrist marker trajectory. Note that during interval $1-2$, the wrist marker shows the last step of the walk towards the target and the beginning of reaching (2) with the paw in stance position. B) Z axis marker temporal displacements. The vertical lines represent the start or the end of movements. $\mathrm{Z}$ axis: vertical axis. Colours and numbers as in A. The displacements of the two markers are temporally aligned so that we can see the displacement of both markers during movements at the same time. C) markers' tangential velocity profiles on the horizontal/depth/vertical (XYZ) axes. Velocity is reported as an absolute value. Colours, numbers and vertical lines as in B. Note that the 
nose marker velocity profile presents many small peaks (below $400 \mathrm{~mm} / \mathrm{sec}$ ), whereas the wrist marker velocity profile present two peaks above $1000 \mathrm{~mm} / \mathrm{sec}$, the left one corresponding to the last step and the right one to the reaching movement. Moreover, the wrist marker does not show a clear velocity peak after reaching i.e. during the grasping movement.
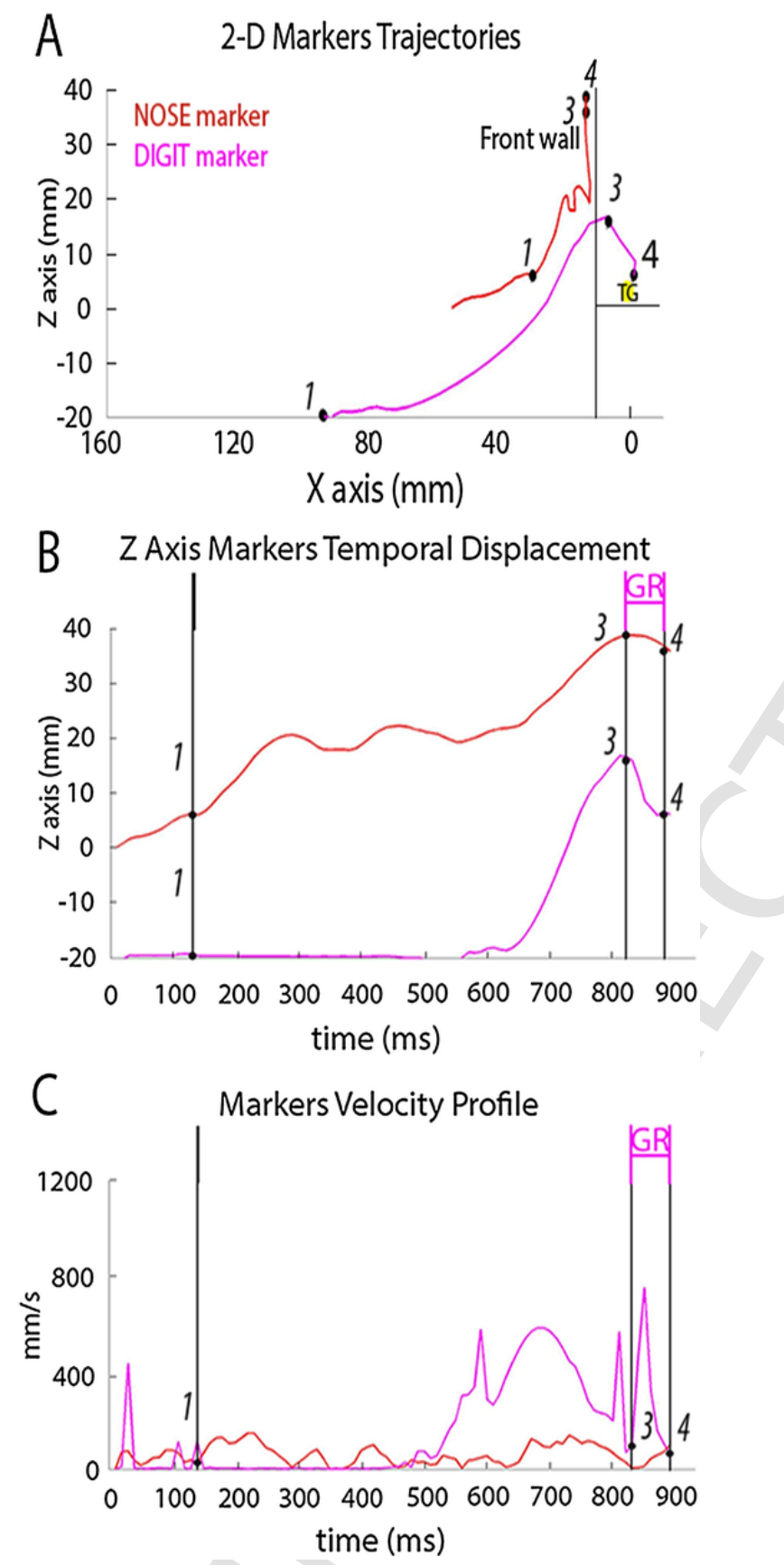

Fig. 4. Example of nose and digit marker trajectories in a trial recorded by Qualisys and split off-line. The figure shows the nose and digit marker trajectories represented as in Fig. 3A. Nose trajectory in red, digit trajectory in pink. GR: grasping movement. Numbers: 1) orienting start point, 3) grasping start point, 4) grasping end point. Orienting end/ reaching start was not derived from the digit marker trajectory. Note that the digit marker comes into contact with the target during grasping (interval 3-4). B) markers' temporal displacements on vertical axis. Vertical lines and axes 0 as in Fig. 3A. C) markers' tangential velocity profiles on horizontal/depth/vertical (XYZ) axes. Colours, numbers and vertical lines as in B. Velocity value as in Fig. 3C. Note that the digit marker velocity profile presents a clear velocity peak during grasping movement. from the left side of the figure to the right: orienting (interval 1-2), reaching (interval 2-3) and grasping (interval 3-4). The simultaneous temporal displacement of the nose and wrist markers along the vertical axis during movements are reproduced in Fig. 3B, and the instantaneous movement velocity profiles, calculated by MATLAB custom program, are shown in Fig. 3C. For both orienting and reaching, onset was defined as the time-point (in ms) at which the tangential velocity exceeded $5 \%$ of the maximum velocity (Fig. $3 \mathrm{C}$ ). The movement velocity was the Euclidean distance between consecutive points divided by the time between frames. End of orienting corresponds to the reaching starting time-point, as defined by the wrist marker (point 2); the end of reaching cannot be defined from the grasping velocity peak, since the wrist marker does not show a clear velocity peak during grasping (see farthest right interval 3-4 in Fig. 3C). For this reason the reaching end was defined as the time-point of minimum distance between the wrist marker and target (point 3). Fig. 4 shows the trajectories on the horizontal and vertical axes, the temporal displacement on the vertical axis, and the velocity peak of the nose (red) and digit (pink) markers. Here the grasping movement is clearly observable, together with its velocity peak (grasping: pink trace interval 3-4 in Fig. 4). Grasping onset was defined as the time-point (in $\mathrm{ms}$ ) at which the tangential velocity exceeded $5 \%$ of maximum velocity, and grasping end was defined as the time-point of minimum velocity of the digit marker before the beginning of the retract movement (point 4 in Fig. 4). Here the finger marker displacement during reaching resulted from the combined limb/digit movement, and consequently faithfully followed neither the limb movement nor the digit movement until the end of reaching i.e. the start of grasping (point 3 in Fig. 4).

\subsection{Data presentation and statistical analysis}

In order to analyse the movement kinematics, for each rat, trajectories were plotted in a 3D reference frame (Fig. 5), and start/end point distributions are presented as horizontal/depth coordinates (Fig. 6). To quantify the starting point distributions along the axes in all animals, values are collectively shown in box plots in Fig. 7. Each movement is identified by four kinematic variables: 1 , movement trajectory length (in $\mathrm{mm}$ ); 2 , movement duration (in $\mathrm{msec}$ ); 3 , mean velocity (in $\mathrm{mm} / \mathrm{s}$ ); and 4, peak velocity (in mm/s) (see Table 1). In Figures 8, 9, 10 and 12, data are plotted as 3D and single or average 2D trajectories and normalized speed profile averages.

To determine if wrist/paw position at the end of reaching tended to occupy the same spatial position as the nose at the end of orienting, first we present two frames corresponding to Poke end and reaching end, respectively (Fig. 11A and B); we also plotted the cumulative normalized displacements of the nose marker during orienting and wrist marker during reaching on the horizontal, depth and vertical axes separately and then in three dimensions (Fig. 11C-F).

To verify whether orienting and reaching end points affected the trial outcome, for each animal we plotted the distribution of the nose marker at orienting end and the distribution of the wrist marker at reaching end of unsuccessful trials (Fig. 11G-M) vs. successful trials (Fig. 11G1-M1).

\subsection{Statistical analysis}

We used build-in custom-made scripts within MATLAB (MathWorks; Natick, MA) and R language (www.R-project.org/) for data analysis, and for the construction of figures and statistical tests. Data are represented as medians and their interquartile ranges of $n$ determinations or MAD (Median absolute deviation). For kinematic parameters, a Kruskal-Wallis test by ranks, followed by Dunn's post-hoc test, were used to determine statistically significant differences between experi- 


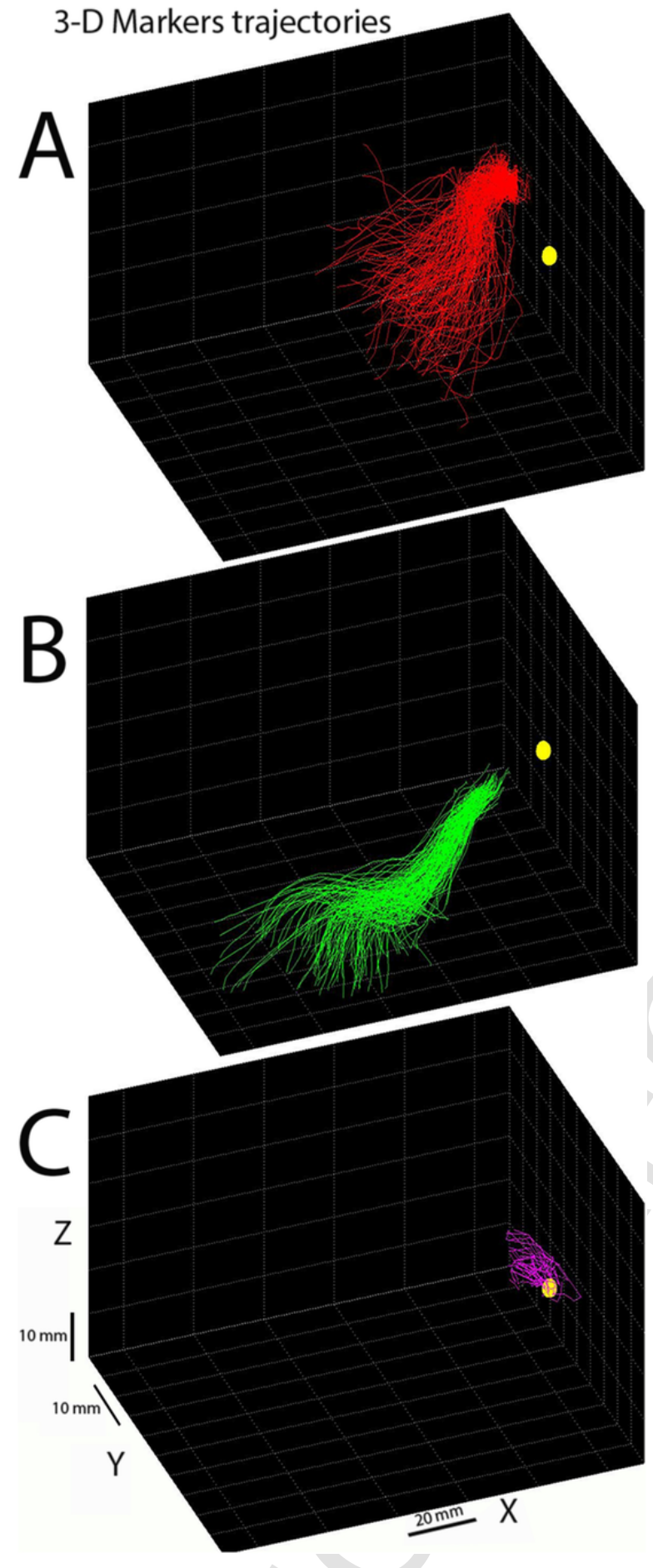

Fig. 5. 3D trajectory reconstruction in the rat 6 . 3D-coordinates system origin $(0,0,0)$ corresponded to the centre of target (yellow sphere). A: orienting (red, $n=78$ ); B: reaching (green, $\mathrm{n}=78$ ); C: grasping (pink, $\mathrm{n}=33$ ). The variability in trajectories and movement start points is appreciable.

mental group values. For all tests a significance level of $\mathrm{p}<0.05$ was set.

\section{Results}

\subsection{Movement sequence description: video-recording vs. 3D recording}

Head and forelimb movement components during skilled reaching were identified by analysing the video recordings frame by frame (Fig.
1, frames A-E on the left) and 3D marker displacement, as captured by Qualysis System (Fig. 1, frames A1-E1 on the right). The task began when the rat walked from the back to the front wall of the box with a cyclical motion of the limbs, a head-down posture and exploratory whisking, until the whiskers came into contact with the front wall (Parmiani et al., 2018). Immediately after the whiskers touched the front wall ( $\sim 20 \mathrm{~ms}$, unpublished data), the orienting movement began with the approach of the head to the front wall and ended at the start of reaching, with the nose poking through the slot to sniff the pellet (Fig. $3 \mathrm{~A}$ and $\mathrm{B}$, red trace $1-2$ ). During orienting, the rat takes the last walking step and makes postural adjustments before the start of the reaching movement (Fig. 3A and B, green trace 1-2). Once the rat located the food, the reaching began with the preferred forelimb lifting from the floor and being directed through the slot towards the pellet (Fig. 3A and $\mathrm{B}$, green trace 2-3). As rat begins reaching, the head is carried upward to allow the paw to cross the slot while the nose stays inside it (Fig. 3A and B, red trace 2-3). Grasping starts at the end of reaching, and ends before the beginning of the withdrawal; it consists of the digits closing to embrace the pellet (Fig. 4 A-C, pink trace 1-2). Therefore, as shown in Figs. 3 and 4, our quantitative analysis takes into account a sequence of three movements: i) orientation to locate the pellet, ii) reaching, or forelimb transport phase, and iii) grasping the pellet. We did not consider the retraction movement (see Methods).

\subsection{Orienting kinematics}

All kinematic values for orienting were derived from the nose marker displacement, which is distinguishable throughout the entire movement (Fig. 1). Table 1 reports the median and IQR of trajectory length and duration, and peak and mean velocities for each animal and population data. All kinematic parameters show a relatively high inter- and intra-subject variability, as demonstrated by the IQR range. In each rat there were direct relationships between trajectory length and duration (r ranging from 0.62 to $0.79, P=0.000$ ), and between trajectory length and peak velocity ( $\mathrm{r}$ ranging from 0.31 to $0.69, P<0.05$ ). In contrast, there was a direct relationship between trajectory length and mean velocity in only three out of five rats ( $\mathrm{r}$ ranging from 0.33 to $0.50, P<0.05$ vs. 0.14 to $0.28, P>0.05$ ). The $3 \mathrm{D}$ reconstruction of all trajectories for each rat shows smooth and continuous trajectories whose length and shape vary according to the position of the start point relative to the target (Fig. 5A). The 2D distribution of start/end points on the horizontal/depth and horizontal/vertical axes shows that for each rat the start points are dispersed, while the end points are focused toward the target (Fig. 6A and C, empty and filled red triangles, respectively). Quantitative analysis of the orienting starting point distribution on the horizontal, depth and vertical axes in all rats is presented in Fig. 7A, which reveals that on the horizontal axis the median starting point value was $41.06 \mathrm{~mm}$ (median values in individual rats range from 39.65 to $42.07 \mathrm{~mm}$ ), and the IQR was $8.07 \mathrm{~mm}$ (median values in individual rats range from 12.61 to $5.06 \mathrm{~mm}$ ); on the depth axis, the median start point was $-0.12 \mathrm{~mm}$ (median values in individual rats range from -1.01 to $5.08 \mathrm{~mm}$ ), and the IQR was $21.95 \mathrm{~mm}$ (median values in individual rats range from 27.88 to $13.82 \mathrm{~mm}$ ); on the vertical axis, the median start point was $0.36 \mathrm{~mm}$ (median values in individual rats range from -8.76 to $10.28 \mathrm{~mm}$ ), and the IQR was $13.54 \mathrm{~mm}$ (median values in individual rats range from 10.5 to $5.24 \mathrm{~mm}$ ). It is clear that all horizontal axis values are positive, and are less dispersed than values on the other axes; the median along the depth axis coincides with zero (target position), with values symmetrically displaced with respect to it; and, finally, the median along the vertical axis close to zero is due to the fact that in two animals the median value was negative, while in the other two it was around zero and in the last animal it was positive. 


\section{2-D distribution of start/end points of OR, RC and GR movements}
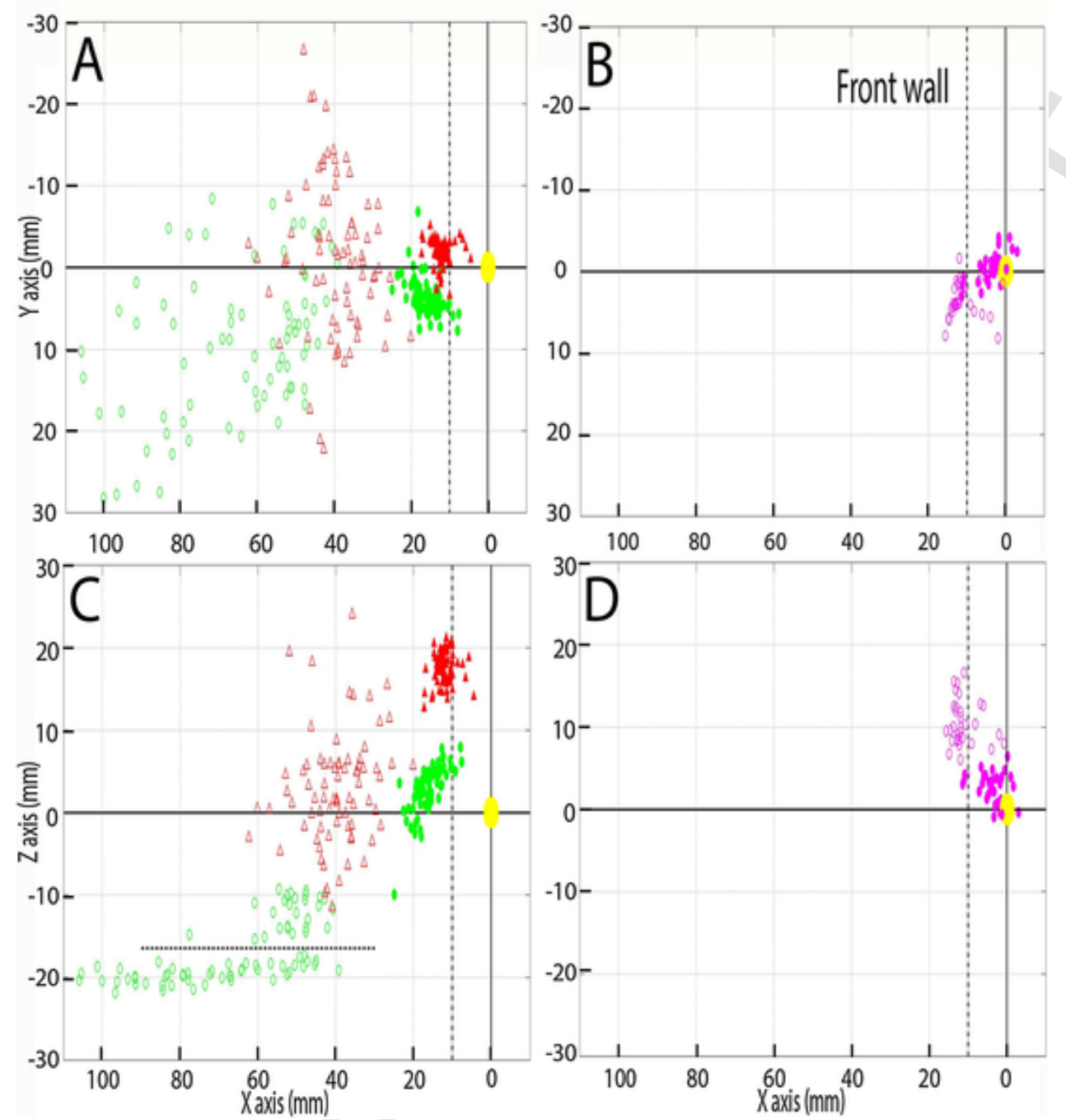

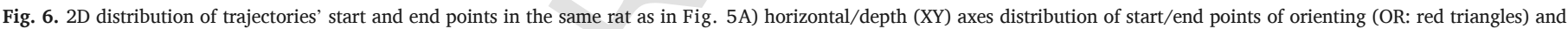

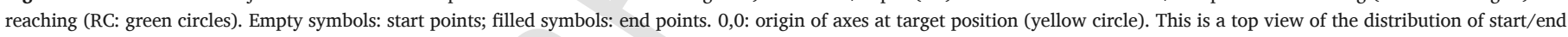

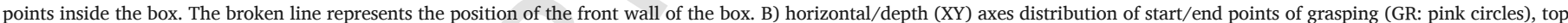

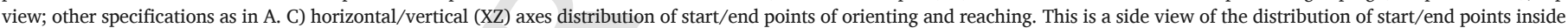

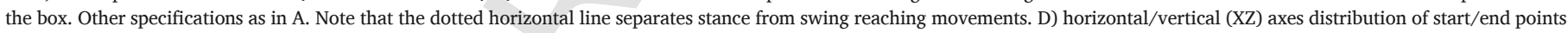
of grasping, side view; other specifications as in A and B.

The frame-by-frame video-analysis of the orienting movement highlights three successive steps: head approaching (HA) toward the front wall, localizing the slot (SL) by touching the nose to the front wall with small head adjustment movements, and poking (PK) the nose through the slot to sniff the pellet (Fig. 1; Parmiani et al., 2018). However, in some trials, the animal arrived close to the front wall and directly inserted its nose into the slot without head adjustments. Taking into account all rats, $83.08 \%$ of orienting trajectories presented all three steps, while in the remaining $16.92 \%$, HA was followed directly by PK (Fig. 8A vs. B). The orienting trajectories with three steps showed significantly greater length and duration than the trajectories that did not present the SL step (long trajectories, median length: $62.05 \mathrm{~mm}$, MAD: $12.95 \mathrm{~mm}$, vs. short trajectories, median length: $49.96 \mathrm{~mm}$, MAD: $9.34 \mathrm{~mm}, \mathrm{p}=0.0000$; long trajectories: median duration: $440 \mathrm{~ms}$, MAD: $100 \mathrm{~ms}$, vs. short trajectories, median duration: $260 \mathrm{~ms}$, MAD: $85 \mathrm{~ms}$, $\mathrm{p}=0.0000$, Wilcoxon rank test). Fig. 8 shows an example of long and short trajectories (A vs. B) and their respective velocity profiles on the horizontal, depth and vertical axes (C vs. D). The long trajectory (Fig.
8A) presents HA (interval 1-2), with major displacements on the horizontal and vertical axes; followed by SL (interval 2-3), with smaller successive displacements along the horizontal, depth and vertical axes; and then PK (interval 3-4), with a displacement mainly along the horizontal axis. The short trajectory does not present the SL step (Fig. 8B). As highlighted by velocity profiles on the horizontal, depth, and vertical axes (Fig. 8C and D), HA onset (point 1) corresponds to orient onset, and its end is the time-point of first minimum velocity on the horizontal axis (point 2), i.e. the point where the nose marker has arrived close to the front wall. SL is characterized by small velocity oscillations along the horizontal, depth and vertical axes between HA and PK (point 2). PK onset is defined as the time-point (in ms) at which the tangential velocity exceeds $5 \%$ of the last velocity peak on the horizontal axis (point 3), and its end corresponds to the orienting end point (point 4). Figs. 8E and F shows that these steps are characterized by different duration and velocity values: SL is the longest-lasting step in long trajectories $(\mathrm{p}=0.0000$, Kruskal-Wallis test by ranks, followed by Dunn's post hoc test), while HA is the step presenting the highest ve- 


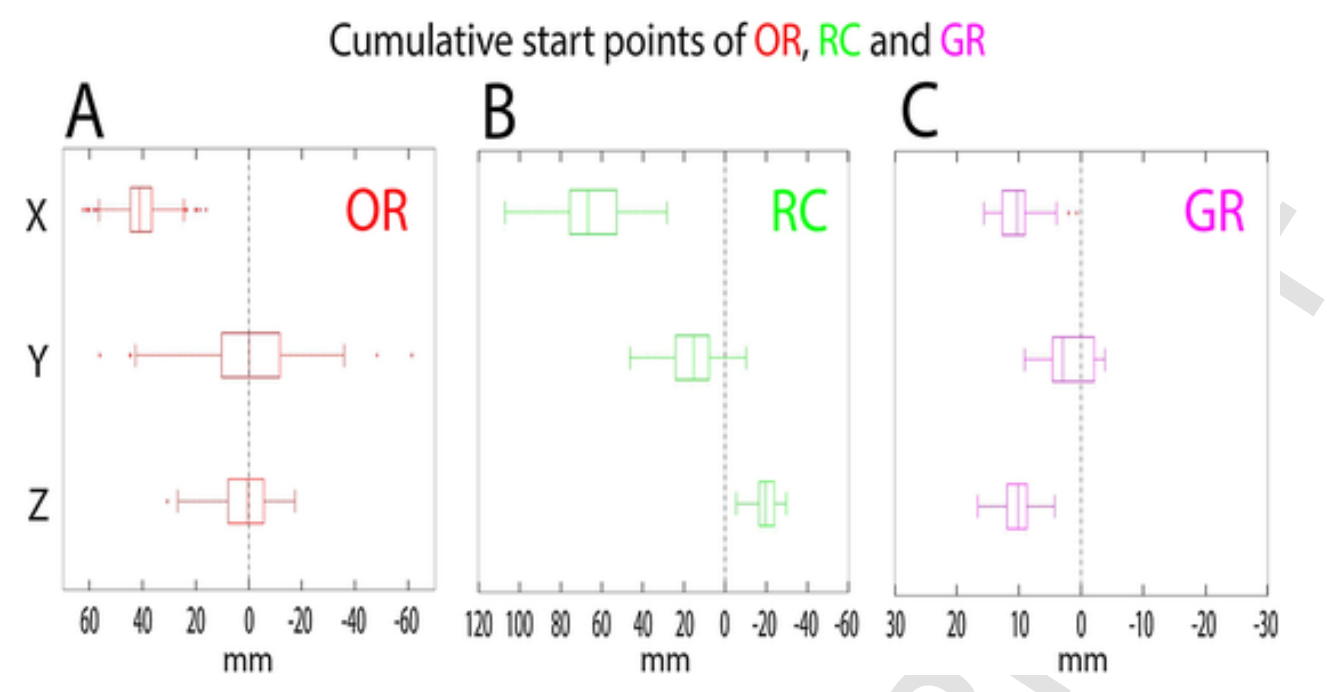

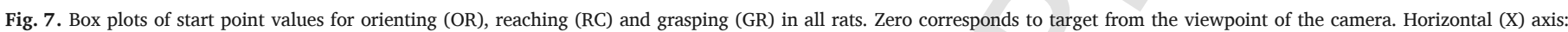

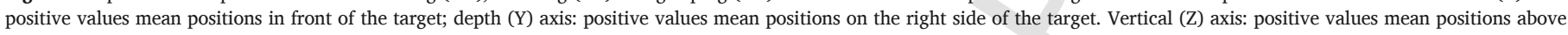

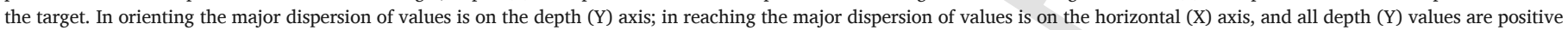
since all animals were right-handed; in grasping, depth $(\mathrm{Y})$ values are distributed around 0.

locity when compared to SL and PK ( $\mathrm{p}=0.0000$, Kruskal-Wallis test by ranks, followed by Dunn's post hoc test).

\subsection{Reaching kinematics}

All kinematic values for reaching are derived from the wrist marker displacement, which is well distinguished throughout the entire movement (Fig. 1). A featuring example of the wrist marker trajectory during reaching is illustrated in the Fig. 3A (green trace: 2-3): in this example the movement starts about $60 \mathrm{~mm}$ along the horizontal axis (2), and ends just beyond the slot, about $10 \mathrm{~mm}$ above the target (3). The wrist marker displacement on the vertical axis versus time also shows the maximum elevation at the end of reaching (Fig. 3B, 3 on green trace). The tangential velocity profile is bell-shaped, with one predominant peak, which is always higher than the predominant peak velocity in the orienting movement (Fig. $3 \mathrm{C}$ green trace $2-3$ vs. red trace $1-2$ ). Table 1 reports the median of trajectory length and duration, and peak and mean velocities for each animal and population data. All kinematic parameters show a relatively high inter- and intra-subject variability, as demonstrated by the interquartile range (IQR, Table1, numbers in italics). In each rat there is a direct relationship between trajectory length and duration ( $\mathrm{r}$ ranging from 0.64 to $0.81, P=0.000$ ), trajectory length and mean velocity ( $\mathrm{r}$ ranging from 0.22 to $0.49, P<0.05$ ) and peak velocity ( $r$ ranging from 0.34 to $0.53, P<0.05$ ).

The 3D trajectory reconstruction, performed in each rat, shows a characteristic pattern; all trajectories appeared smooth and continuous with variable length and shape as a function of the position of the starting point relative to the target (Fig. 5B). To evaluate spatial distribution of the start/end points of reaching trajectories, a 2D representation of all start/end points on the horizontal/depth and horizontal/vertical planes were plotted for each rat. As can be seen from the example in Fig. 6A and $\mathrm{C}$ (horizontal/depth and horizontal/vertical axes distributions, respectively, green circles), the start points of reaching are dispersed (Fig. 6A and $\mathrm{C}$, empty green circles); conversely, all end points are focused towards the target and are localized in front of it, mostly at the inner side of the front wall, owing to the position of the wrist marker (Fig. 6A and $\mathrm{C}$, filled green circles).

A quantitative analysis of reaching start point distribution on the horizontal, depth and vertical axes in all rats is presented in Fig. 7B, which shows that the median start point on the horizontal axis was
$66.61 \mathrm{~mm}$ (median values in individual rats range from 68.99 to $60.52 \mathrm{~mm}$ ), with an IQR of $22.69 \mathrm{~mm}$ (median values in individual rats range from 29.64 to $12.54 \mathrm{~mm}$ ); the median start point on the depth axis was $15.22 \mathrm{~mm}$ (median values in individual rats range from 24.40 to $6.01 \mathrm{~mm}$ ), IQR $16.30 \mathrm{~mm}$ (median values in individual rats range from 16.28 to $8.38 \mathrm{~mm}$ ); and the median start point on the vertical axis was $-19.80 \mathrm{~mm}$ (median values in individual rats range from -24.97 to $-16.74 \mathrm{~mm}$ ), IQR $7.47 \mathrm{~mm}$ (median values in individual rats range from 9.99 to $2.15 \mathrm{~mm}$ ). In all rats, horizontal axis values were positive and more dispersed than those on the other axes; the depth axis values were also positive, since all animals were right-handed, and those on the vertical axis were negative, since the start points were below the target position.

In Fig. 6C, we can discern two different bimodal reaching start point distributions, one along the horizontal axis and the other along the vertical axis. Along the horizontal axis, in relation to the distance from the target, we can distinguish between far and near start points i.e. beyond or within the first quartile from the target (see Fig. 7B). Based on this, long and short reaching trajectories may be analysed separately (Fig. 9A vs. B: long trajectories, median: $76.35 \mathrm{~mm}$, MAD: $7.81 \mathrm{~mm}$, vs. short trajectories, median: $49.36 \mathrm{~mm}$, MAD: $5.86 \mathrm{~mm}, \mathrm{p}=0.0000$, Wilcoxon rank test), as they are also characterized by different durations (long trajectories, median: $200 \mathrm{~ms}$, MAD: $30 \mathrm{~ms}$, vs. short trajectories, median: $140 \mathrm{~ms}$, MAD: $20 \mathrm{~ms}, \mathrm{p}=0.0000$, Wilcoxon rank test). Long trajectories are characterized by an intermediate phase of wrist/paw advancement (one velocity peak on the X axis: Fig. 9A2) between two phases of elevation (two velocity peaks on the vertical axis: Fig. 9A3). Both velocity peaks on the vertical axis are approximately three times lower than those on the $\mathrm{X}$ axis. Short trajectories, on the other hand, are characterized by a single phase of wrist elevation and advancement toward the target with a single peak on the horizontal and vertical axes, occurring at around $40 \%$ of the normalized duration, and the velocity peak on the vertical axis is half the size of the peak on the horizontal axis (Fig. 9B2 and B3).

In relation to the vertical axis distribution, within short trajectories (Fig. 6C) we can distinguish two modalities of reach start, depending on the position of the paw in that precise moment, i.e. in the stance or swing phase of the last step. Quantitative analysis reveals that $53 \%$ of swing trajectories were short trajectories, and of these, $79 \%$ are distributed above the value of $-18 \mathrm{~mm}$ along the vertical axis. This observa- 
Table 1

Kinematic parameters values for Orienting, Reaching and Grasping movements.

\begin{tabular}{|c|c|c|c|c|c|c|c|c|c|c|c|c|c|c|c|c|c|c|}
\hline & \multicolumn{6}{|c|}{ Orienting } & \multicolumn{6}{|c|}{ Reaching } & \multicolumn{6}{|c|}{ Grasping } \\
\hline & R1 & R3 & R6 & R5 & R4 & GROUP & R1 & R3 & R6 & R5 & R4 & GROUP & R1 & R3 & R6 & R5 & R4 & GROUP \\
\hline & $S=3$ & $S=5$ & $S=5$ & $S=7$ & $S=3$ & $\mathrm{~S}=23$ & $\mathrm{~S}=3$ & $S=5$ & $S=5$ & $S=7$ & $S=3$ & $\mathrm{~S}=23$ & $S=2$ & $S=2$ & $S=3$ & & & $S=7$ \\
\hline & $\mathrm{n}=47$ & $\mathrm{n}=86$ & $\mathrm{n}=77$ & $\mathrm{n}=150$ & $\mathrm{n}=56$ & $\mathrm{n}=416$ & $\mathrm{n}=47$ & $\mathrm{n}=86$ & $\mathrm{n}=77$ & $\mathrm{n}=150$ & $\mathrm{n}=56$ & $\mathrm{n}=416$ & $\mathrm{n}=16$ & $\mathrm{n}=9$ & $\mathrm{n}=33$ & & & $\mathrm{n}=58$ \\
\hline \multirow[t]{2}{*}{ Trajectory Length (mm) } & 52.47 & 51.49 & 56.26 & 74.81 & 71.17 & 63.26 & 78.30 & 71.66 & 68.07 & 73.91 & 62.65 & 70.84 & 15.49 & 22.86 & 19.82 & / & / & 19.27 \\
\hline & 22.29 & 11.27 & 19.02 & 19.18 & 25.65 & 25.18 & 26.05 & 15.28 & 28.78 & 24.38 & 27.62 & 24.33 & 3.01 & 4.93 & 6.90 & & & 8.41 \\
\hline \multirow[t]{2}{*}{ Duration (ms) } & 280 & 530 & 400 & 590 & 520 & 495 & 170 & 220 & 170 & 170 & 230 & 185 & 50 & 80 & 60 & / & / & 60 \\
\hline & 145 & 150 & 120 & 200 & 210 & 225 & 50 & 60 & 70 & 70 & 115 & 75 & 30 & 30 & 30 & & & 20 \\
\hline \multirow[t]{2}{*}{ Max speed (mm/s) } & 426.83 & 289.35 & 430.86 & 361.73 & 340.51 & 359.58 & 976.18 & 750.18 & 779.16 & 979.28 & 530.83 & 815.49 & 568.59 & 545.67 & 745.58 & / & / & 656.73 \\
\hline & 170.20 & 85.28 & 144.71 & 140.16 & 134.12 & 160.61 & 257.17 & 177.64 & 276.89 & 353.14 & 172.43 & 347.96 & 140.22 & 319.90 & 228.22 & & & 301.17 \\
\hline \multirow[t]{2}{*}{ Mean speed (mm/s) } & 162.93 & 88.37 & 128.19 & 117.12 & 125.70 & 118.56 & 435.31 & 304.78 & 346.23 & 378.28 & 240.29 & 340.39 & 243.16 & 264.98 & 314.71 & / & / & 280.51 \\
\hline & 55.68 & 17.36 & 32.08 & 22.76 & 44.40 & 43.14 & 94.96 & 55.64 & 86.16 & 98.61 & 87.50 & 113.16 & 116.13 & 110.30 & 90.55 & & & 103.27 \\
\hline
\end{tabular}




\section{3-D NOSE Marker Trajectories}
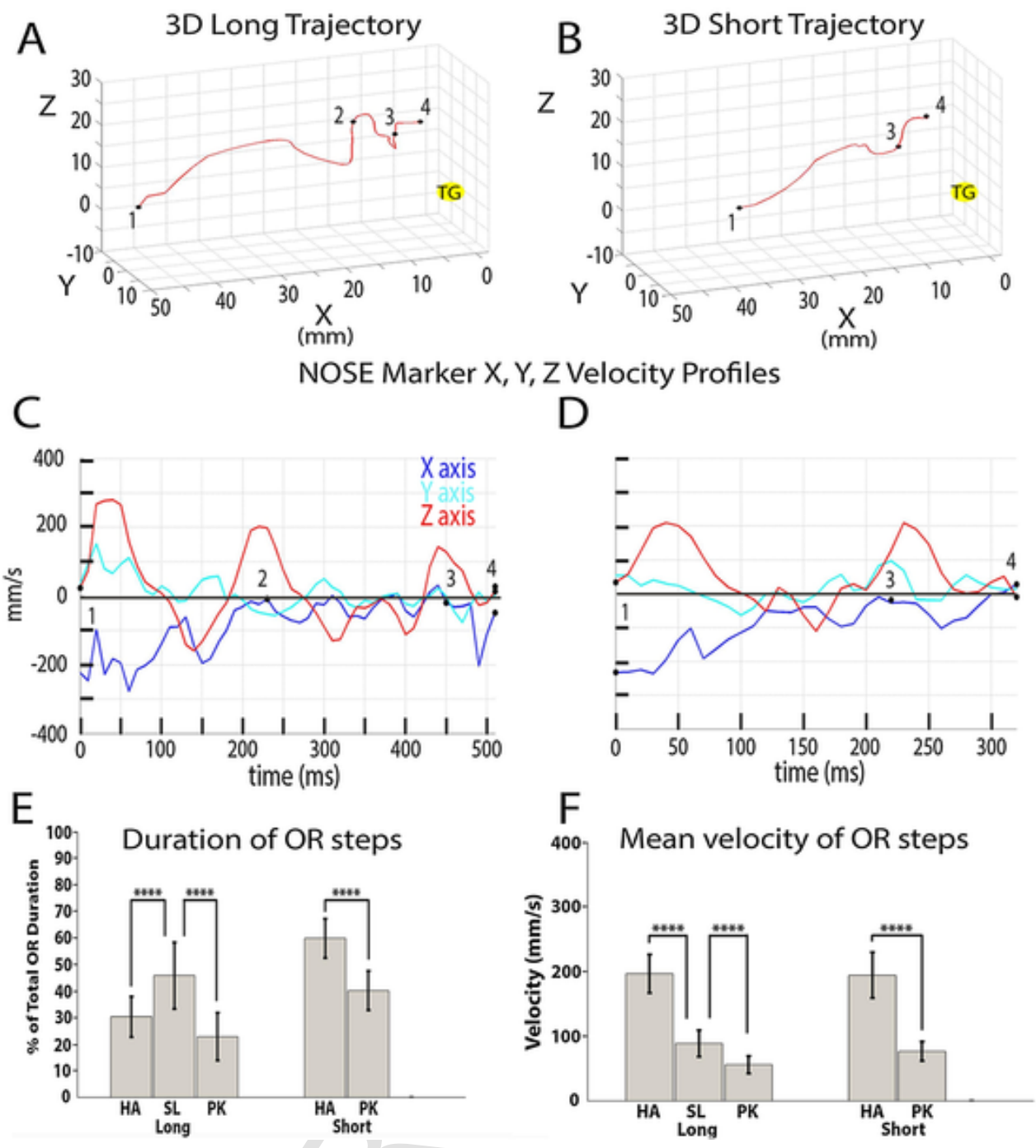

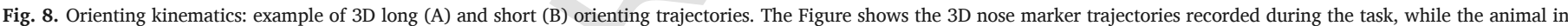

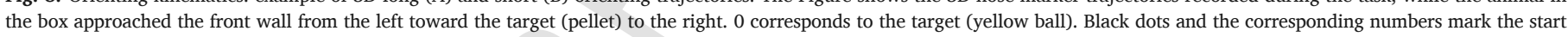

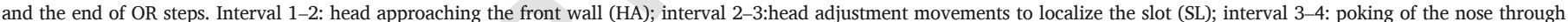

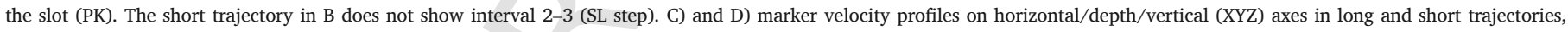

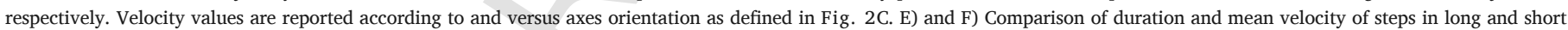
trajectories. (**** $\mathrm{p}=0.0000$, Kruskal-Wallis test by ranks, followed by Dunn's post hoc test).

tion stands for a segregation within short reach starting point along vertical axis. Averaging the stance and swing trajectories in relation to the normalized trial duration (Fig. 10) shows that reaching starts correspond to the last step in the stance position in panel A, and to the last step in the swing position in panel B. Moreover, Fig. 10 reveals that stance reaching starts at around $80 \%$ of the normalized orienting-reaching duration, while swing reaching starts at around $70 \%$ of the normalized orienting-reaching duration.

Video-recording analysis highlighted that the wrist/paw position at the end of reaching tended to occupy the same spatial position as the nose at the end of orienting (Fig. 11A and B). To verify this observation, we plotted the cumulative normalized displacements of the nose marker during orienting, and the wrist marker during reaching on the horizontal, depth and vertical axes separately (Fig. 11C-E), and then as 3D coordinates (Fig. 11F). Horizontal, depth and 3D plots clearly show a convergence of nose and wrist marker displacements at the end of the orienting and reaching movements, while the vertical plot shows a parallel displacement of the two markers due to the their anatomical position.

Finally, we were interested to determine any differences in orienting and reaching spatial end point distributions in successful trials. To this end we plotted the orienting and reaching end point distribution, first from unsuccessful trials (Fig. 11G-M ) and then only from successful trials, (Fig. 11G1-M1) in each animal. This data highlighted that the wrist/paw positions of successful trials tended to occupy spatial positions that were closer to the target. Kruskal-Wallis test comparing 3D orienting and reaching end points distributions in unsuccessful vs. successful trials revealed a significant effect only for reaching (Kruskal-Wallis test: orienting Z score $=-1.6534, P=0.098$; reaching $\mathrm{Z}$ score $=-4.5094 ; P=0.0000$ ).

\subsection{Grasping kinematics}

All kinematic values of grasping are derived from the digit marker displacement, which is distinguishable throughout the entire movement 


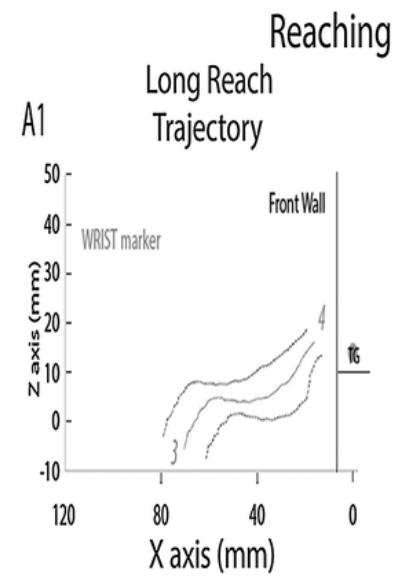

Kinematics
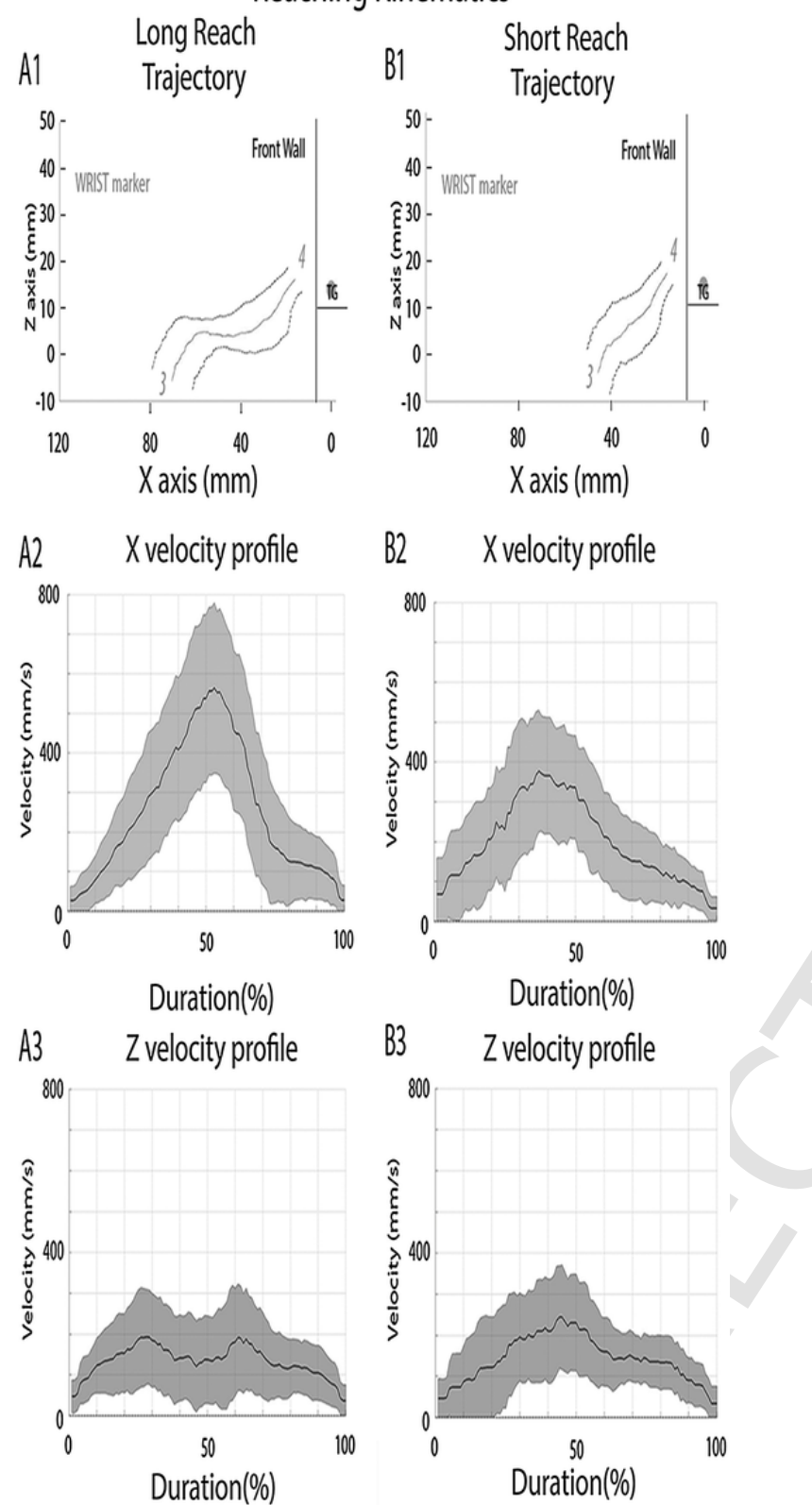

Fig. 9. Reaching kinematics: long and short trajectories. Reaching cumulative averaging of long (A) and short (B) trajectories expressed as median \pm MAD. Lines and axes 0 as in Fig. 3A. A2) and B2) normalized velocity profile on horizontal (X) axis. A3) and B3 normalized velocity profile on vertical $(\mathrm{Z})$ axis. Note the different trajectory shapes and velocity profiles, depending on the distance of start points from target.

(Fig. 1). A featuring example of digit marker displacement during grasping is illustrated in the Fig. 4A (dark pink trace: $3-4$ ): in this example the movement starts beyond the slot, at about $25 \mathrm{~mm}$ from the horizontal axis 0 (3), ends just over the target (4), and presents a clear single velocity peak (Fig. 4C, dark pink trace: 3-4). The 3D reconstruction of all trajectories in each animal (Fig. 5C) and the 2D representation of all start/end points on the horizontal/depth and horizontal/vertical planes (Fig. 6B and D) confirm the pattern shown in Fig. 4. Fig. $7 \mathrm{C}$, on the other hand, reveals that the median value of grasping start points on the horizontal axis was $10.28 \mathrm{~mm}$ (median values in individual rats range from 12.25 to $9.42 \mathrm{~mm}$ ), with an IQR of $3.67 \mathrm{~mm}$ (median values in individual rats range from 2.7 to $1.21 \mathrm{~mm}$ ); the median value of start points on the depth axis was $2.95 \mathrm{~mm}$ (median values in individual rats range from -3.37 to 5.71 ), IQR $6.71 \mathrm{~mm}$ (ranging in a single rat from 1.12 to $2.60 \mathrm{~mm}$ ); and the median value of start points on the vertical axis was $10.14 \mathrm{~mm}$ (median values in individual rats range from 11.90 to $9.11 \mathrm{~mm}$ ), IQR $3.20 \mathrm{~mm}$ (median values in individual rats range from 4.32 to $1.90 \mathrm{~mm}$ ). It is clear that median grasping values on all axes are positive, and the IQR along the depth axis is distributed around zero, even though not symmetrically so.

Table 1 reports the median grasping trajectory length and duration, and peak and mean velocities for each animal and population data. All kinematic parameters show a relatively high inter- and intra-subject variability, as shown by the interquartile range (IQR, Table 1, numbers in italics). In all rats there was a direct relationship between trajectory length and both mean velocity ( $\mathrm{r}$ ranging from 0.65 to $0.87, P<0.05$ ) and peak velocity ( $\mathrm{r}$ ranging from 0.65 to $0.83, P<0.05$ ), while in two out of three rats there was a direct relationship between trajectory length and duration ( $\mathrm{r}$ ranging from 0.59 to $0.62, P=0.0103$ ); in one rat there was an inverse relationship, but this did not reach significance ( $\mathrm{r}=-0.62$ to $0.62, P=0.0767$ ).

Fig. 12 shows the averaging of trajectories and velocity profiles along horizontal and vertical axes for the grasping movement. Trajectory averaging confirmed the lowering and approaching of the marker toward the target (Fig. 12A1). The averaged velocity profile showed a velocity peak on the horizontal axis that was twice as high as that in vertical axis, occurring at around $20-30 \%$ of the normalized grasping duration (Fig. 12A2and A3). Since the marker applied to the digits reduced the successful pellet-grasping score far below the $50 \%$, we did not look for a difference between hit and miss trials.

\section{Discussion}

The method provides insight into the motor organization of skilled movement in the rat, highlighting the orienting-to-food behaviour, the relationship between wrist movement and nose movement, and features of the grasp. To value if the markers impede the normal movements we considered the success percentage of trials execution with and without markers. Markers applied to the rat's body did not impede the normal orienting and reaching movements, except the digit marker, which reduced the successful pellet-grasping score to $<50 \%$.

Using three high-speed infrared digital cameras to track the entire expected range motion of marker movements in a large capture volume, we obtained a 3D recording of full skilled reaching kinematics in a rat moving freely in the reaching box, which was previously unavailable. 3D kinematics were analysed by Qualisys Track Manager software and MATLAB, a set-up designed to be reliable, high-performance and easy to use, which measures the distance between two constantly visible markers rotating in the capture volume. Using this set-up, tracking can be done in real-time, with minimal latency, or in post-processing. Qualisys trajectory editing made it easy to identify, edit and process trajectories and detect artefacts. This approach undoubtedly represents an improvement with respect to high-speed single camera recording (Wong et al., 2015; Ellens et al., 2016; Nica et al., 2018; Parmiani et al., 2018), but Qualysis, as all passive optical systems, is limited by its inability to measure motion when markers move within $2 \mathrm{~mm}$ of each other, as well as a high set-up cost. Although the new approach involving machine-learning algorithms such as DeepLabCut presents higher resolution and greater degrees of freedom at a single digit level than our method, it requires time-consuming digitization of joint segments for markerless videorecordings (Mathis et al., 2018; Bova et al., 2019). Our method, on the other hand, required significantly reduced time to label data, and obviates the need for off-line manual marking of videos. Furthermore, it provides an efficient means of capturing volumes containing the entire range of marker movements in the rat. Finally, our methods may also be transferable to other tasks as 
Wrist Marker Trajectory during OR and RC
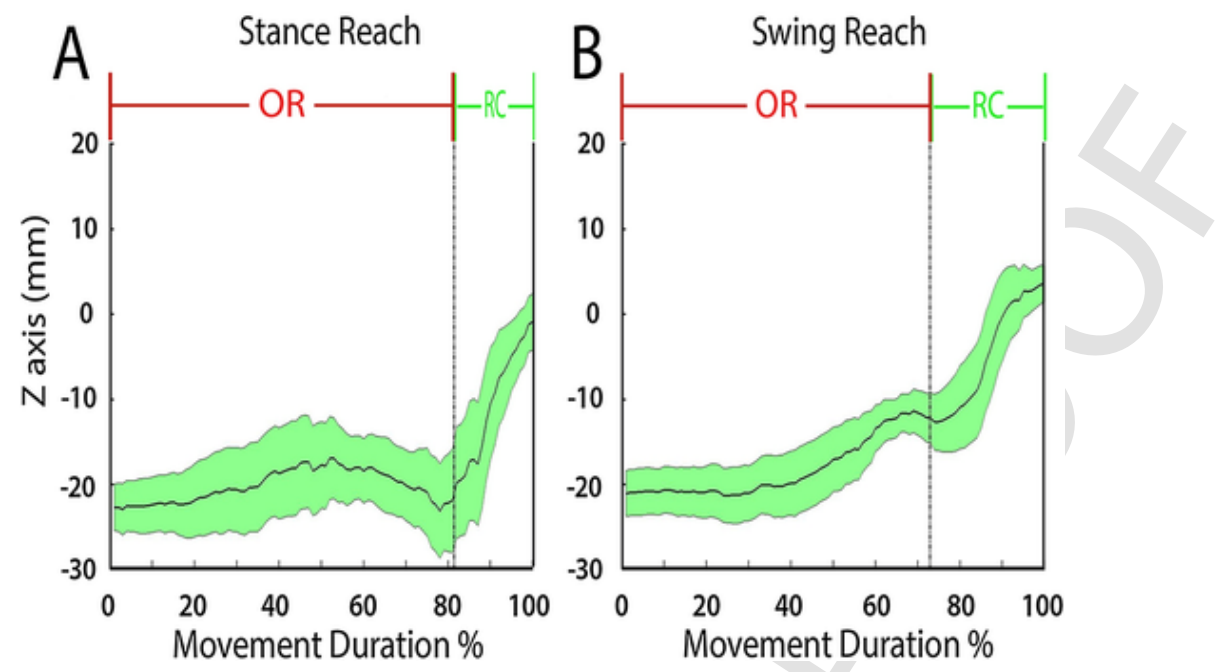

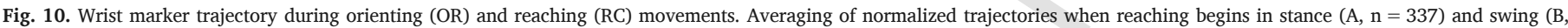

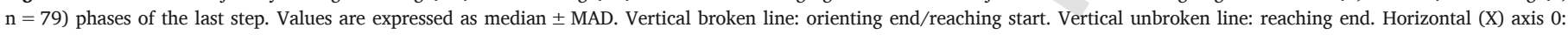
orienting start; vertical (Z) axis 0: target position.

the isometric pull task (Hays et al,2013) and the staircase task (Montoya et al., 1991) or could be used with fully-automated (Fenrich et al., 2016) and semi-automated (Torres-Espin et al., 2018) versions of the skilled reaching and grasping task. For this purpose it is necessary to comply with two operational conditions; firstly, the space around the box must be free enough to allow the markers to be visible to all the cameras during the entire movement; and secondly, for a good markers detection, reflective plastic or metal elements should not be present within the cameras field or need to be masked.

\subsection{Orienting movement}

The orienting movement comprises the coordination of different body parts, including the eyes, ears, head, and trunk. In our experiments, orienting was considered to comprise the head movement after the macrovibrissae contact the front wall, followed by slot localization and the nose poke through it (Parmiani et al., 2018). Comparing kinematics values for orienting vs. reaching movements, orienting was characterized by a slightly shorter trajectory length but longer duration, while maximum and mean speeds were lower than reaching values. Both behavioural and kinematic analyses highlighted that orienting movement was composed of three successive steps: HA, SL and PK. Triggered by macrovibrissae collision with front wall, HA was rapid, with head displacement until the snout contacted the front wall. Then, SL, guided by repetitive snout/microvibrissae tactile exploration of the front wall, was characterized by slow, small head displacements around the slot. Finally, PK was a slow, small movement as the nose approached the pellet along the horizontal axis. These data suggested that all of these three steps involved in orienting could be feedback-guided, and that olfactory, somatosensory and proprioceptive input could be integrated in specific ways in the spatio-temporal development of each step (Whishaw and Tomie, 1989; Parmiani et al., 2018).

\subsection{Reaching movement}

Our kinematic analysis showed that reaching movements were characterized by smooth trajectory displacement and bell-shape velocity profiles. Compared to the orienting movement, reaching showed similar trajectory length, but with half the duration and double the speed.
This suggested that reaching was less controlled by sensory feedback than orienting, and presented a ballistic nature (Jeannerod, 1990; Whishaw and Karl, 2014). In our set-up, the object/target size and position were fixed, and the reaching began at various distances from the target, allowing us to evaluate trajectories of different amplitude. Distance effects were significant for trajectory length, shape and duration, as well as the amplitude and number of velocity peaks, and the percentage of time to peak velocity. These kinematics parameters did not support the principle of "isochrony", according to which the movement velocity, depending on the amount of distance to cover, produces a constant duration of movement (Viviani and McCollum, 1983). However, in the rat, skilled reaching is a conditioned behaviour, constrained in a box, and is therefore not comparable to the primate's reaching behaviour in a naturalistic setting, where isochrony is the rule (Sartori et al., 2013a, 2013b).

Our kinematic results highlight two different modalities of starting the reach, namely in the stance or swing phase of the last step. The observation that the swing reaching appeared as a single continuous and uniform movement that smoothly shifted from step to reach further supports the hypothesis that reaching is derived from an evolution of the step (Karl and Whishaw, 2013).

Interestingly, our findings showed that the wrist/paw position at the end of the reaching movement tended to occupy the same spatial position as the nose at the end of the orienting, and this relationship was a fixed spatial link in the present context. We therefore propose that in single pellet reaching within dedicated box, where the rat poked through the slot to identify the pellet before reaching it, the nose works as pointing system for wrist/paw positioning. Alternatively, another possibility is that the constrains of the task force the nose and paw to occupy the same space. Moreover, when considering the orienting and reaching end point distributions in unsuccessful vs. successful trials, we noted that the orienting distribution did not substantially change, while the reaching distribution lacked points more distant from the target. This suggested that unsuccessful reaching was explained not by the orienting end-point positions, but rather by the reaching end-point positions. 
OR and RC end points
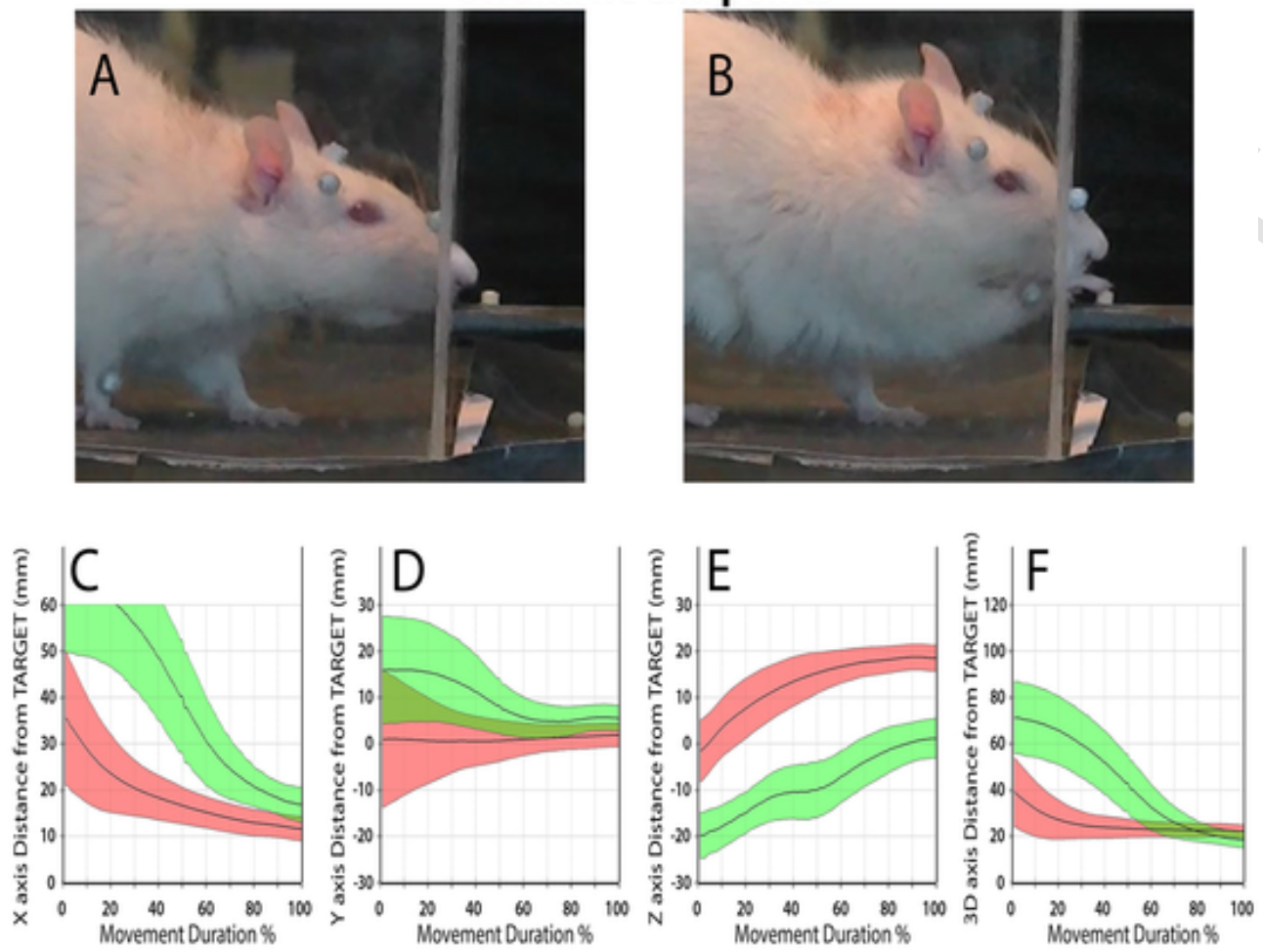

\section{OR and RC end points: unsuccessful trials}

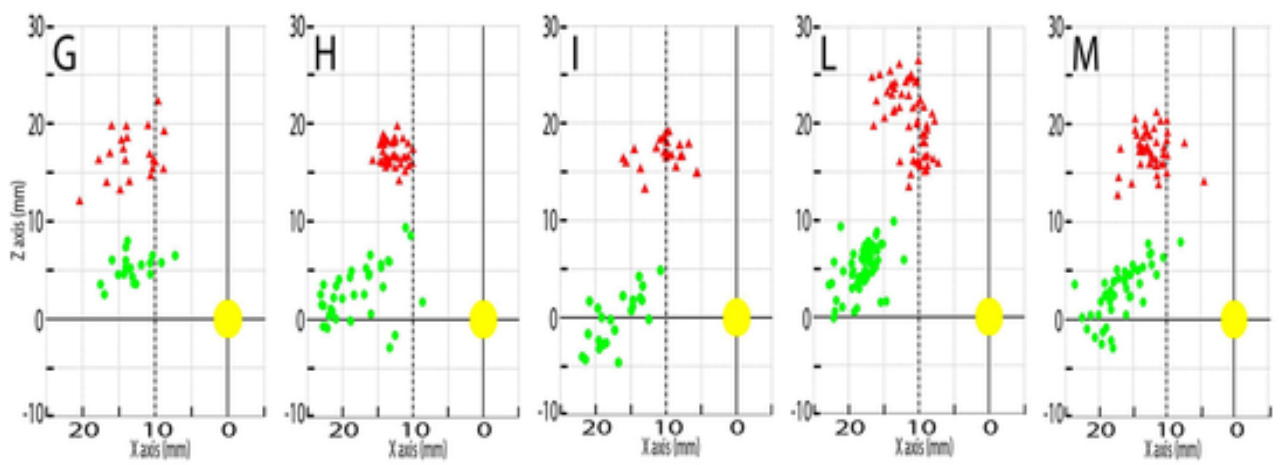

OR and RC end points: successful trials
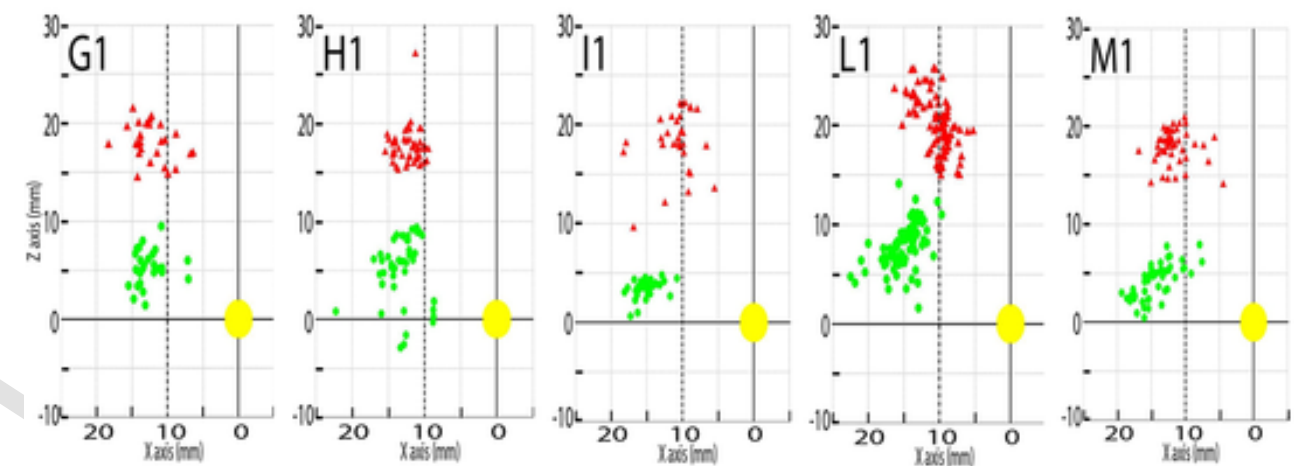

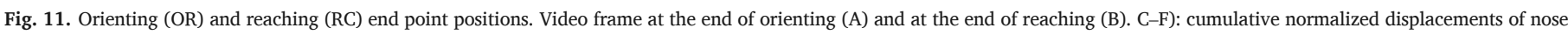

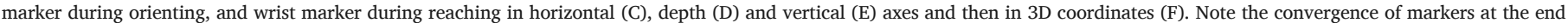

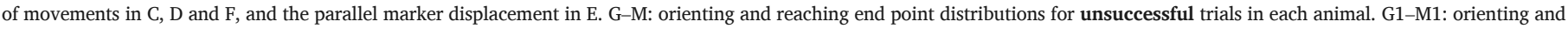
reaching end point distributions for successful trials in each animal. Note that wrist/paw positions in successful trials tended to be closer to the target. 


\section{Grasping Kinematics}
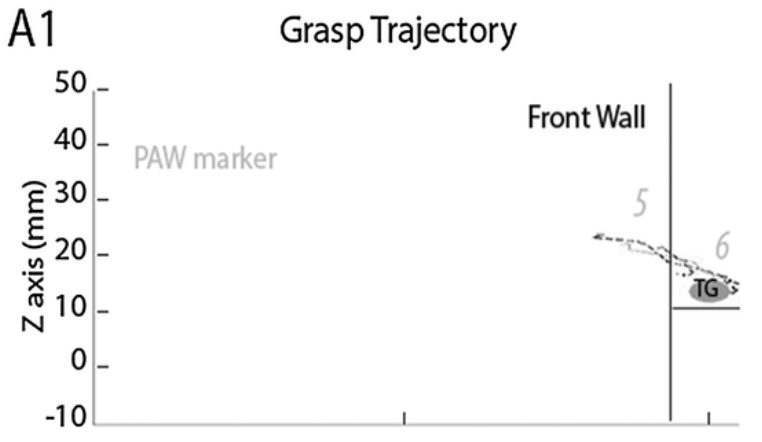

60

30

0

\section{$X$ axis $(\mathrm{mm})$}

A2

$X$ velocity profile

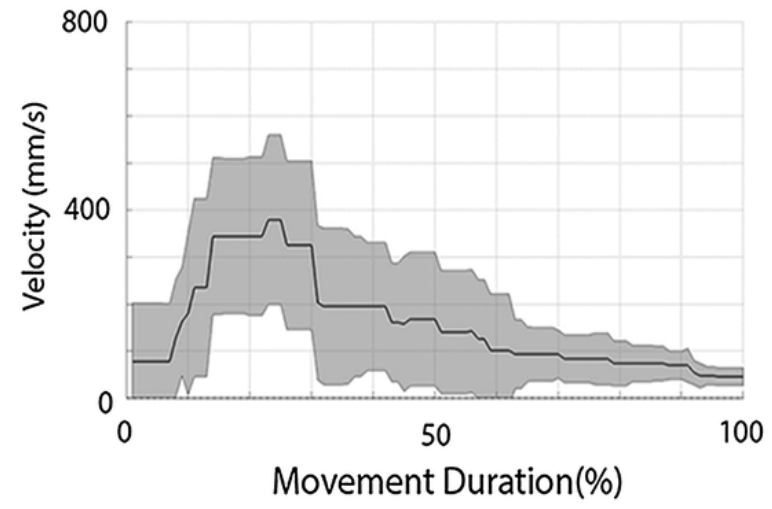

\section{A3 Z velocity profile}

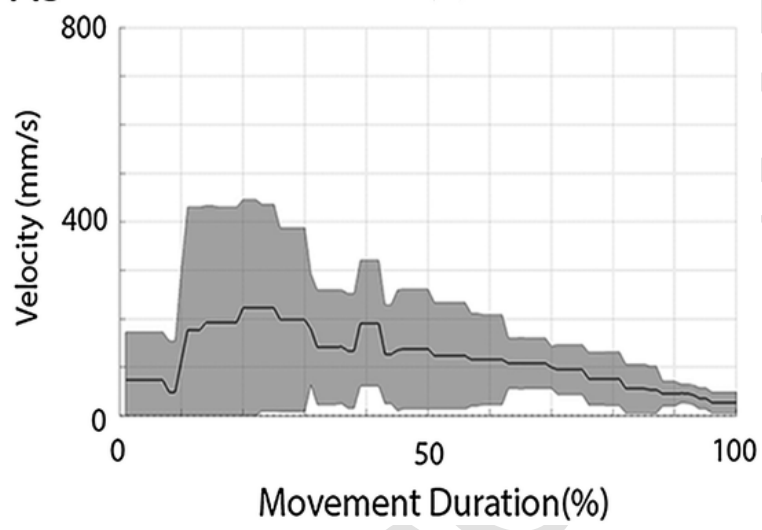

Fig. 12. Grasping kinematics. Cumulative averaging of grasping trajectories (A) expressed as median \pm MAD. Lines and axes 0 as in Fig. $3 \mathrm{~A}$. B: normalized velocity profile on horizontal (X) axis. C: normalized velocity profile on vertical (Z) axis. Note that velocity peak falls between the first and second quarter of the normalized movement duration.

\subsection{Grasping movement}

Primates employ four grip types: side, precision, thumb-to-second/ third, and power grip (Macfarlane and Graziano, 2009). In the rat, the grip corresponds to the primate's power grip or whole hand prehension (Metz and Whishaw, 2000; Alaverdashvili et al., 2008) using digits $2-5$ to grasp the pellet, according to the final position of the paw on the shelf (Whishaw et al., 2010). This was the reason why in these experiments we chose to fix the marker at the last phalangeal joint of the two middle digits. This position enabled us to pre- sent data related to the phase of paw closing, when the digits were in maximum aperture, at the end of reaching and before the start of the retracting movement. As such, grasping was characterized by a distinct bell-shaped speed profile, and comparable kinematic values among the three animals recorded. Comparing kinematics values of grasping vs. reaching movements, grasping was characterized by the shortest trajectory length and the lowest duration, while maximum and mean speeds were slightly lower than reaching values.

\subsection{Conclusions}

In summary, the present set-up used an infrared three-dimensional motion-tracking system, which does not required significant time and effort to manually mark videos, coupled with behavioural video-recordings. This method allowed us to simultaneously record the movements of the wrist and nose markers, and separately the movements of the digit marker. By off-line reconstruction and analysis of these marker trajectories, we identified kinematic criteria for defining the beginning and the end of each movement which proved to classify the movement components effectively in all the animals studied.

\section{Acknowledgments}

This work was supported by a local grant awarded by the University of Ferrara, and a grant awarded by the Ministry of Education, Universities and Research (PRIN-2015, n. F52F16000840001).

\section{References}

Alaverdashvili, M., Leblond, H., Rossignol, S., Whishaw, I.Q., 2008. Cineradiographic (video X-ray) analysis of skilled reaching in a single pellet reaching task provides insight into relative contribution of body, head, oral, and forelimb movement in rats. Behav. Brain Res. 192, 232-247.

Azim, E., Jiang, J., Alstermark, B., Jessell, T.M., 2014. Skilled reaching relies on a V2a propriospinal internal copy circuit. Nature 508, 357-363.

Bova, A., Kernodie, K., Mulligan, K., Leventhal, D., 2019. Automated rat single-pellet reaching with 3-Dimensional reconstruction of paw and digit trajectories (2019, in press). J Vis Exp JoVE.

Ellens, D.J., Gaidica, M., Toader, A., Peng, S., Shue, S., John, T., Bova, A., Leventhal, D.K. 2016. An automated rat single pellet reaching system with high-speed video capture. J. Neurosci. Methods 271, 119-127.

Gharbawie, O.A., Whishaw, I.Q., 2006. Parallel stages of learning and recovery of skilled reaching after motor cortex stroke: "oppositions" organize normal and compensatory movements. Behav. Brain Res. 175, 249-262.

Hermer-Vazquez, L., Hermer-Vazquez, R., Chapin, J.K., 2007. The reach-to-grasp-food task for rats: a rare case of modularity in animal behavior? Behav. Brain Res. 177, $322-328$.

Jeannerod, M., 1990. The Neural and Behavioural Organization of Goal-directed Movements, Clarendon Press; Oxford University Press, Oxford [England]; New York.

Karl, J.M., Whishaw, I.Q., 2013. Different evolutionary origins for the reach and the grasp: an explanation for dual visuomotor channels in primate parietofrontal cortex. Front. Neurol. 4, 208.

Klein, A., L-AR, S., Whishaw, I.Q., Dunnett, S.B., 2012. The use of rodent skilled reaching as a translational model for investigating brain damage and disease. Neurosci. Biobehav. Rev. 36, 1030-1042.

Macfarlane, N.B.W., Graziano, M.S.A., 2009. Diversity of grip in Macaca mulatta. Exp. Brain Res. 197, 255-268.

Mathis, A., Mamidanna, P., Cury, K.M., Abe, T., Murthy, V.N., Mathis, M.W., Bethge, M., 2018. DeepLabCut: markerless pose estimation of user-defined body parts with deep learning. Nat. Neurosci. 21, 1281-1289.

Metz, G.A., Whishaw, I.Q., 2000. Skilled reaching an action pattern: stability in rat (Rattus norvegicus) grasping movements as a function of changing food pellet size. Behav. Brain Res. 116, 111-122.

Nica, I., Deprez, M., Nuttin, B., Aerts, J.-M., 2018. Automated assessment of endpoint and kinematic features of skilled reaching in rats. Front. Behav. Neurosci. 11, 255.

Parmiani, P., Lucchetti, C., Franchi, G., 2018. Whisker and nose tactile sense guide rat behavior in a skilled reaching task. Front. Behav. Neurosci. 12, 24

Sartori, L., Camperio Ciani, A., Bulgheroni, M., Castiello, U., 2013. Reaching and grasping behavior in Macaca fascicularis: a kinematic study. Exp. Brain Res. 224, 119-124.

Sartori, L., Camperio-Ciani, A., Bulgheroni, M., Castiello, U., 2013. Reach-to-grasp movements in Macaca fascicularis monkeys: the Isochrony Principle at work. Front. Psychol. 4, 114.

Viaro, R., Bonazzi, L., Maggiolini, E., Franchi, G., 2017. Cerebellar modulation of cortically evoked complex movements in rats. Cereb. Cortex 1991 (27), 3525-3541.

Viviani, P., McCollum, G., 1983. The relation between linear extent and velocity in drawing movements. Neuroscience 10, 211-218.

Whishaw, I.Q., 1996. An endpoint, descriptive, and kinematic comparison of skilled reaching in mice (Mus musculus) with rats (Rattus norvegicus). Behav. Brain Res. 78, $101-111$. 
Whishaw, I.Q., Dringenberg, H.C., Pellis, S.M., 1992. Spontaneous forelimb grasping in free feeding by rats: motor cortex aids limb and digit positioning. Behav. Brain Res. 48, 113-125.

Whishaw, I.Q., Faraji, J., Kuntz, J.R., Mirza Agha, B., Metz, G.A.S., Mohajerani, M.H., 2017. The syntactic organization of pasta-eating and the structure of reach movements in the head-fixed mouse. Sci. Rep. 7, 10987.

Whishaw, I.Q., Karl, J.M., 2014. The contribution of the reach and the grasp to shaping brain and behaviour. Can. J. Exp. Psychol. Rev. 68, 223-235.

Whishaw, I.Q., Pellis, S.M., 1990. The structure of skilled forelimb reaching in the rat: a proximally driven movement with a single distal rotatory component. Behav. Brain Res. 41, 49-59.
Whishaw, I.Q., Tomie, J.A., 1989. Olfaction directs skilled forelimb reaching in the rat. Behav. Brain Res. 32, 11-21.

Whishaw, I.Q., Travis, S.G., Koppe, S.W., Sacrey, L.-A., Gholamrezaei, G., Gorny, B., 2010 Hand shaping in the rat: conserved release and collection vs. flexible manipulation in overground walking, ladder rung walking, cylinder exploration, and skilled reaching. Behav. Brain Res. 206, 21-31.

Whishaw, I.Q., Whishaw, P., Gorny, B., 2008. The structure of skilled forelimb reaching in the rat: a movement rating scale. J. Vis. Exp. JoVE..

Wong, C.C., Ramanathan, D.S., Gulati, T., Won, S.J., Ganguly, K., 2015. An automated behavioral box to assess forelimb function in rats. J. Neurosci. Methods 246, 30-37. 